\title{
A geometric approach to cut-generating functions
}

\author{
Amitabh Basu* $\quad$ Michele Conforti ${ }^{\dagger} \quad$ Marco Di Summa ${ }^{\dagger}$
}

November 17, 2018

\begin{abstract}
The cutting-plane approach to integer programming was initiated more that 40 years ago: Gomory introduced the corner polyhedron as a relaxation of a mixed integer set in tableau form and Balas introduced intersection cuts for the corner polyhedron. This line of research was left dormant for several decades until relatively recently, when a paper of Andersen, Louveaux, Weismantel and Wolsey generated renewed interest in the corner polyhedron and intersection cuts. Recent developments rely on tools drawn from convex analysis, geometry and number theory, and constitute an elegant bridge between these areas and integer programming. We survey these results and highlight recent breakthroughs in this area.
\end{abstract}

\section{Introduction}

The cutting-plane approach to integer programming (IP) was initiated in the early 1970s with the works of Gomory [47, 49, 55, 48, 50, 51, Gomory and Johnson [52, 53, and Johnson [58] on the corner polyhedron, and of Balas [11] on intersection cuts generated from convex sets. Their approach aimed at the development of a theory of valid inequalities (to be used as cuts) for integer programs, pure or mixed, independently of the structure and the data of the problem on hand. Gomory introduced a universal model which provided a relaxation for any integer program defined by constraints in tableau form and studied cut-generating functions. These functions when applied to a specific IP problem, provide a valid inequality that is not satisfied by the basic solution associated with the tableau.

While the point of view of Gomory was algebraic, the approach of Balas was essentially based on the geometry of the sets to be studied. As an example, split cuts are the simplest and most effective family of intersection cuts. They are equivalent to Gomory's mixed integer (GMI) cuts, which are generated by applying a cut-generating function to a single row of a tableau, see e.g. Theorem 5.5 in 34].

Possibly inspired by the deep and elegant results in combinatorial optimization and polyhedral combinatorics, research in IP then shifted its focus on the study of strong (facetdefining) valid inequalities for structured integer programs of the combinatorial type, mostly 0,1 programs.

\footnotetext{
${ }^{*}$ Department of Applied Mathematics and Statistics, The Johns Hopkins University, MD, USA

${ }^{\dagger}$ Dipartimento di Matematica, Università degli Studi di Padova, Italy. Supported by the grant "Progetto di Ateneo 2013" of the University of Padova.
} 
Renewed interest in Gomory's approach was recently sparked by a paper of Andersen, Louveaux, Weismantel and Wolsey [4]. They define a relaxation of Gomory's model whose tableaux has two rows. This is a 2-dimensional model and can be represented in the plane. They show that, besides nonnegativity constraints, the facet-defining inequalities are naturally associated with splits (a region between two parallel lines), triangles and quadrilaterals whose interior does not contain an integer point. This allows one to derive valid inequalities by exploiting the combined effect of two rows, instead of a single row.

The extension of this model to any dimension (i.e., number of integer variables) was pioneered by Borozan and Cornuéjols [27] and by Basu, Conforti, Cornuéjols and Zambelli [17]. The main finding is that like in the 2-dimensional case, facet-defining inequalities are naturally associated with full-dimensional convex sets whose interior does not contain an integer point. Furthermore these sets are polyhedra. In this survey we highlight the importance of this fact as it provides a simple formula to compute the associated cut-generating function.

Indeed, Lovász [63] stated that maximal convex sets whose interior does not contain an integer point are polyhedra, but the first proof appears in [17], and an alternate proof can be found in [8]. These proofs use the simultaneous approximation theorem of Dirichlet and Minkowski's convex body theorem. So important results from number theory and convex geometry are fundamental in proving polyhedrality, which is essential to get a computable formula for the cut-generating function. We highlight the use of these and other "classical" theorems in this survey.

This survey first introduces in Section 2 a general mixed-integer set which provides a framework to study cut-generating functions. We then highlight in Section 3 some areas of applicability of this general mixed-integer set.

The next three sections are essentially devoted to special cases of this general mixedinteger set. Section 4 deals with the case introduced by Andersen et al. 4]. We first explain how the gauge function, from convex analysis, links convex sets whose interiors do not contain integer points and valid inequalities. We then give a novel and concise proof of a Lovász's characterization of maximal convex sets whose interior does not contain an integer point. It is based on a proposition that characterizes the sets arising as a projection of the integers on a subspace. We then survey extensions of these results to a more general setting that includes complementarity and nonlinear constraints.

Section 5 considers the special case of the mixed-integer set with only integer variables. This case, first introduced by Gomory and Johnson in [52, 53], was the starting point of the theory of cut-generating functions and is also known as the infinite group relaxation in the literature. We show recent progress in extending classical results beyond the single-row problem to arbitrary number of rows; in particular, we emphasize the use of the Knaster-KuratowskiMazurciewicz lemma, a classical result from fixed-point theory, in this development.

In Section 6, we discuss the general set with both continuous and integer variables. We focus on the aspect of lifting, where the quest for computable formulas for cutting planes leads to unexpected connections with the theory of tilings and coverings of Euclidean space. Such questions have classically been considered within the field of geometry of numbers and the recent connection with integer programming leads to a rich theory. We also highlight a recent discovery from [25] that topological arguments, such as the classical Invariance of Domain theorem from algebraic topology, can lead to important results in this area. 
To summarize, in this survey we concentrate on results from the cut-generating function approach to cutting planes. An introduction to this topic can be found in Chapter 6 of [34]; see also [32]. There is a parallel body of work which uses finite cyclic groups to study Gomory's corner polyhedron and cutting planes. Richard and Dey's survey [66] on the group approach covers this aspect, as well as its links with cut-generating functions. Further, there has been lot of work in studying closures of families of cutting planes, and convergence issues in cutting plane algorithms. We do not discuss these topics in this survey; the reader is referred to the survey by Del Pia and Weismantel 39. A recent survey by Basu, Köppe and Hildebrand $[22$ delves deeper into aspects of cut-generating functions for the pure integer case that are discussed in Section 5 of this survey.

We believe that the recent results that we survey here, such as the computable formula for cut-generating functions derived in Section 4 , together with the theory of lifting discussed in Section 6. are the first steps towards making cut-generating functions in general dimensions a viable computational tool. Prior to this, only one-dimensional cut-generating functions were explicitly provided with which one could perform computations.

In fact, even the one-dimensional theory developed by Gomory did not find its way into IP solvers for decades and was believed to be of little or no computational use. This point of view changed dramatically in the mid 1990s following the work of Balas, Ceria, Cornuéjols, Natraj [12]; see [35] for a recent account. Today most cutting planes currently implemented in software are based on this one-dimensional theory, such as GMI cuts from tableau rows, mixed integer rounding inequalities and lift-and-project cuts [34]. We hope that the corresponding progress for higher-dimensional cut-generating functions can provide another boost to the efficiency of mixed-integer optimization solvers. On the flip side, this poses greater challenges in choosing the "right" cutting planes, since this theory significantly increases the pool of available cuts. Computational experiments have been conducted by Dey, Lodi, Tramontani, Wolsey [40], Basu, Bonami, Cornuéjols, Margot [15], and Louveaux and Poirrier [62], based mostly on the special case discussed in Section 4. However, some of the developments surveyed here have not been computationally tested, and the effectiveness of these new findings remains open.

\section{Separation and valid functions}

For fixed $n \in \mathbb{N}$, let $S$ be a closed subset of $\mathbb{R}^{n}$ that does not contain the origin 0 . In this survey, we consider subsets of the following form:

$$
X_{S}(R, P):=\left\{(s, y) \in \mathbb{R}_{+}^{k} \times \mathbb{Z}_{+}^{\ell}: R s+P y \in S\right\}
$$

where $k, \ell \in \mathbb{Z}_{+}, R \in \mathbb{R}^{n \times k}$ and $P \in \mathbb{R}^{n \times \ell}$ are matrices. We allow $k=0$ or $\ell=0$, but not both. These sets were first introduced by Johnson in [58] and [59], based on earlier work by Gomory and Johnson in [52, 53]. We address the following

Separation problem: Find a closed half-space that contains $X_{S}(R, P)$ but not the origin.

The fact that $S$ is closed and $0 \notin S$ implies 0 is not in the closed convex hull of $X_{S}(R, P)$ [31, Lemma 2.1]. Hence such a half-space always exists. 
This problem arises typically when one wants to design a cutting-plane method to optimize a (linear) function over $X_{S}(R, P)$ and has on hand a solution (the origin 0 ) to a relaxation of the problem (see Section 3).

We develop a theory that for fixed $S$ addresses the separation problem independently of $R$ and $P$ by introducing the concept of valid pair.

A valid pair $(\psi, \pi)$ for $S$ is a pair of functions $\psi, \pi: \mathbb{R}^{n} \rightarrow \mathbb{R}$ such that for every choice of $k, \ell, R$ and $P$,

$$
\sum \psi(r) s_{r}+\sum \pi(p) y_{p} \geq 1
$$

is an inequality separating 0 from $X_{S}(R, P)$ (this is the reason for the choice of 1 for the right hand side). We use the convention that the first sum is taken over the columns $r$ of $R$, where $s_{r}$ denotes the continuous variable associated with column $r$; similarly, the second sum ranges over the columns $p$ of $P$, and $y_{p}$ denotes the integer variable associated with column $p$. This convention for summations will be used throughout the paper. Valid pairs are also known as cut-generating pairs, and inequality (2.2) is often called a cut.

When $\ell=0$ in (2.1), i.e., when all variables are continuous, we obtain a set of the type

$$
C_{S}(R):=\left\{s \in \mathbb{R}_{+}^{k}: R s \in S\right\}
$$

where $k \geq 1$. A function $\psi: \mathbb{R}^{n} \rightarrow \mathbb{R}$ is a valid function for $S$ if $\sum \psi(r) s_{r} \geq 1$ is an inequality separating 0 from $C_{S}(R)$ for every $k$ and $R$. Again we use the convention that the above sum is taken over the columns $r$ of $R$. This model, here referred to as the continuous model, will be discussed in Section 4 .

When $k=0$, i.e., when all variables are integer, sets of the form (2.1) become

$$
I_{S}(P):=\left\{y \in \mathbb{Z}_{+}^{\ell}: P y \in S\right\}
$$

where $\ell \geq 1$. A function $\pi: \mathbb{R}^{n} \rightarrow \mathbb{R}$ is an integer valid function for $S$ if $\sum \pi(p) y_{p} \geq 1$ is an inequality separating 0 from $I_{S}(P)$ for every $\ell$ and $P$. This model, here referred to as the pure integer model, will be discussed in Section 5 .

When both $k$ and $\ell$ are positive, we refer to (2.1) as the mixed integer model; this will be discussed in Section 6 .

There is a natural partial order on the set of valid pairs, namely $\left(\psi^{\prime}, \pi^{\prime}\right) \leq(\psi, \pi)$ if and only if $\psi^{\prime} \leq \psi$ and $\pi^{\prime} \leq \pi$. Since $\left\{(s, y): \sum \psi^{\prime}(r) s_{r}+\sum \pi^{\prime}(p) y_{p} \geq 1, s \geq 0, y \geq 0\right\} \subseteq$ $\left\{(s, y): \sum \psi(r) s_{r}+\sum \pi(p) y_{p} \geq 1, s \geq 0, y \geq 0\right\}$ whenever $\left(\psi^{\prime}, \pi^{\prime}\right) \leq(\psi, \pi)$, all the cuts obtained from $(\psi, \pi)$ are dominated by those obtained from $\left(\psi^{\prime}, \pi^{\prime}\right)$. The minimal elements under this partial order are called minimal valid pairs. Similarly, one defines minimal valid functions $\psi$ and minimal integer valid functions $\pi$. An application of Zorn's lemma (see e.g. [24, Theorem 1.1]) shows that every valid pair (resp., valid function, integer valid function) is dominated by a minimal valid pair (resp., minimal valid function, minimal integer valid function). Thus one can concentrate on the minimal valid functions and pairs.

REMARK 2.1. A natural question is whether cut generating functions are sufficient in the following sense: Given a fixed closed set $S \subseteq \mathbb{R}^{n} \backslash\{0\}$ and a fixed pair of matrices $R, P$, 
is the closed convex hull of $X_{S}(R, P)$ described by the intersection of all inequalities of the type (2.2) when we consider all possible minimal valid pairs for $S$ ? The same question can be phrased for the continuous model (2.3), as well as for the pure integer model (2.4).

This question, in its full generality, is not settled. For the continuous model (2.3), Conforti et al. [31, Example 6.1] show that for a particular set $S$ minimal valid functions do not suffice. However, if $S$ is contained in the conical hull of the columns of $R$, Cornuéjols et al. [38] prove that $C_{S}(R)$ is defined by the inequalities derived from cut-generating functions. Earlier Zambelli [71] showed this to be true when $S=b+\mathbb{Z}^{n}$ for some $b \in \mathbb{R}^{n} \backslash \mathbb{Z}^{n}$.

For the pure integer model (2.4), when $n=1$ and $R$ is a rational matrix, an affirmative answer can be deduced from [22, Theorem 8.3] (this result is a restatement of results appearing in [52]).

Notation Given a convex subset $K$ of $\mathbb{R}^{n}$, we denote with $\operatorname{dim}(K), \operatorname{int}(K), \operatorname{relint}(K)$, $\mathrm{cl}(K), \operatorname{aff}(K), \operatorname{rec}(K), \operatorname{lin}(K)$ the dimension, interior, relative interior, topological closure, affine hull, recession cone and lineality space of $K$. These are standard notions in convex analysis, see e.g. 61].

We denote with $B(x, \varepsilon)$ the closed ball of center $x$ and radius $\varepsilon$. Given $V \subseteq \mathbb{R}^{n}$, we indicate with $\operatorname{conv}(V)$ its convex hull and with $\langle V\rangle$ the linear space generated by $V$. Given a linear subspace $L$, we denote by $L^{\perp}$ the orthogonal complement of $L$ and by $\operatorname{proj}_{L}(\cdot)$ the orthogonal projection on $L$.

\section{Significance of the mixed integer set (2.1)}

The model (2.1) contains as special cases several classical optimization models. Some examples are illustrated below.

1. Gomory's relaxation of IP and extensions. The classical way in which model (2.1) arises is as follows; see [50]. Let $x+P y=b$ be the system of equations that defines a (final) tableau of the linear-programming (LP) relaxation of a pure IP problem, whose feasible set is $\left\{(x, y) \in \mathbb{Z}_{+}^{n} \times \mathbb{Z}_{+}^{\ell}: x+P y=b\right\}$.

When $b \in \mathbb{Z}^{n}$, the LP basic solution $x=b, y=0$ is a solution to the IP and is an optimal solution when the tableau is final. When $b \notin \mathbb{Z}^{n}$, a relaxation of the above set can be obtained by dropping the nonnegativity condition on $x$. Thus the feasible set of this relaxation can be expressed only in terms of $y$ as

$$
\left\{y \in \mathbb{Z}_{+}^{\ell}: P y \in b+\mathbb{Z}^{n}\right\} \text {. }
$$

Note that this fits the setting (2.4) where $S=b+\mathbb{Z}^{n}$, and $0 \notin S$ because $b \notin \mathbb{Z}^{n}$. The convex hull of (3.1) is known as the corner polyhedron.

Of course, if $S=b-\mathbb{Z}_{+}^{n}$, i.e. the condition $x \geq 0$ is maintained, no relaxation occurs. This case was one of the main motivations to study sets of the type $S=\left(b+\mathbb{Z}^{n}\right) \cap Q$, where $Q$ is a rational polyhedron, see [18, 43, 70].

The above can be extended to the mixed integer case as follows. Let $x+R s+P y=b$ be the system of equations that defines a (final) tableau of the LP relaxation of a 
mixed integer program, where $s$ is the vector of nonnegative continuous variables, $y$ is the vector of nonnegative integer variables, and $x$ is the vector of basic nonnegative variables, which may be continuous or integer. However if the $x \geq 0$ constraint is relaxed, one may assume that $x$ is a vector of integer variables, as every continuous basic variable is defined by the corresponding equation of the tableau with no further restriction; hence these equations can now be dropped. The model in this case is

$$
\left\{(s, y) \in \mathbb{R}_{+}^{k} \times \mathbb{Z}_{+}^{\ell}: R s+P y \in b+\mathbb{Z}^{n}\right\} .
$$

Andersen, Louveaux, Weismantel and Wolsey [4, Borozan and Cornuéjols [27], and Basu, Conforti, Cornuéjols and Zambelli [17] studied the relaxation of the above model in which the integrality of the nonbasic variables is relaxed: $\left\{s \in \mathbb{R}_{+}^{k+\ell}:(R, P) s \in\right.$ $\left.b+\mathbb{Z}^{n}\right\}$. This important special case, which fits (2.3), will be discussed in Section 4.1. Again, sets $S$ different from $b+\mathbb{Z}^{n}$ may be considered.

2. Mixed integer (structured) convex programs. Mixed integer programming with convex constraints is a powerful generalization of mixed integer linear programming that can model problems in applications with inherent nonlinearities [5, 6, 29, 30, 69]. The classical model here is

$$
\left\{(x, s, y) \in \mathbb{R}^{n} \times \mathbb{R}_{+}^{k} \times \mathbb{Z}_{+}^{\ell}: R s+P y+x=b, \quad x \in K \cap\left(\mathbb{R}^{t} \times \mathbb{Z}^{n-t}\right)\right\}
$$

where $K$ is a convex set. A special case of this model is mixed integer conic programming, where $K$ is taken to be a closed, convex, pointed cone. This framework is readily obtained from (2.1) by setting $S=b-K \cap\left(\mathbb{R}^{t} \times \mathbb{Z}^{n-t}\right)$.

3. Complementarity problems with integer constraints. In such problems, the feasible region consists of all integer points in a given polyhedron $Q=\left\{(x, y) \in \mathbb{R}_{+}^{n} \times \mathbb{R}_{+}^{\ell}: x+\right.$ $P y=b\}$ that satisfy the complementarity constraints $x_{i} x_{j}=0,(i, j) \in E$ where $E$ is a subset of $\{1, \ldots, n\} \times\{1, \ldots, n\}$. This can be modeled using (2.4) by setting $S=b-\left\{x \in \mathbb{Z}_{+}^{n}: x_{i} x_{j}=0,(i, j) \in E\right\}$.

\section{The continuous model}

Given a closed set $S \subseteq \mathbb{R}^{n} \backslash\{0\}$, we study valid functions $\psi: \mathbb{R}^{n} \rightarrow \mathbb{R}$ for the model $C_{S}(R)$, as defined in (2.3). We characterize the valid functions that are minimal. We will see that minimal valid functions for $S$ are naturally associated with maximal $S$-free convex sets. A closed, convex set $K \subseteq \mathbb{R}^{n}$ is $S$-free if $\operatorname{int}(K) \cap S=\emptyset$, and an $S$-free convex set $K$ is maximal if $K$ is not properly contained in any $S$-free convex set. With a straightforward application of Zorn's lemma, it can be shown that every $S$-free convex set is contained in a maximal one 31 .

In Section 4.1 we will consider the case $S=b+\mathbb{Z}^{n}$ for some fixed $b \in \mathbb{R}^{n} \backslash \mathbb{Z}^{n}$. As discussed in Section 3, this case is of particular importance in integer programming. We will then treat the more general case of an arbitrary closed set $S \subseteq \mathbb{R}^{n} \backslash\{0\}$ in Section 4.2. 


\subsection{The case $S=b+\mathbb{Z}^{n}$}

Here we assume $S=b+\mathbb{Z}^{n}$ for a fixed $b \in \mathbb{R}^{n} \backslash \mathbb{Z}^{n}$, hence $0 \notin S$.

We recall some definitions from convex analysis. A function $g: \mathbb{R}^{n} \rightarrow \mathbb{R}$ is positively homogeneous if $g(\lambda r)=\lambda g(r)$ for every $r \in \mathbb{R}^{n}$ and every $\lambda>0$. Note that if $g$ is positively homogeneous, then $g(0)=0$. Indeed, for any $\lambda>0$, we have that $g(0)=g(\lambda 0)=\lambda g(0)$, which implies that $g(0)=0$. A function $g: \mathbb{R}^{n} \rightarrow \mathbb{R}$ is subadditive if $g\left(r^{1}\right)+g\left(r^{2}\right) \geq g\left(r^{1}+r^{2}\right)$ for all $r^{1}, r^{2} \in \mathbb{R}^{n}$. The function $g$ is sublinear if it is both subadditive and positively homogeneous. Note that since sublinear functions are convex, they are continuous in the interior of their domain. The following lemma appears first in [27.

Lemma 4.1. Assume $S=b+\mathbb{Z}^{n}$ for some $b \notin \mathbb{Z}^{n}$, and let $\psi: \mathbb{R}^{n} \rightarrow \mathbb{R}$ be a minimal valid function for $S$. Then $\psi$ is sublinear and nonnegative.

Proof. We first note that $\psi(0) \geq 0$. Indeed, consider any point $\bar{s} \in C_{S}(R)$ for some $n \times k$ matrix $R$ containing the 0 -column. Let $\tilde{s}=\bar{s}$ except for the component $\tilde{s}_{0}$, which is set to an arbitrarily large value $k$. Since $\tilde{s} \in C_{S}(R)$ and $\psi$ is valid, we have that $\sum \psi(r) \tilde{s}_{r}+\psi(0) k \geq 1$. For this inequality to hold for all $k>0$, we must have $\psi(0) \geq 0$.

(a) $\psi$ is sublinear. We first prove that $\psi$ is subadditive. When $r^{1}=0$ or $r^{2}=0$, inequality $\psi\left(r^{1}\right)+\psi\left(r^{2}\right) \geq \psi\left(r^{1}+r^{2}\right)$ follows from $\psi(0) \geq 0$. Assume that for $r^{1}, r^{2} \neq 0, \psi\left(r^{1}\right)+\psi\left(r^{2}\right)<$ $\psi\left(r^{1}+r^{2}\right)$. Set $\psi^{\prime}\left(r^{1}+r^{2}\right)=\psi\left(r^{1}\right)+\psi\left(r^{2}\right)$ and $\psi^{\prime}(r)=\psi(r)$ for $r \neq r^{1}+r^{2}$. Then $\psi^{\prime} \leq \psi$, $\psi^{\prime} \neq \psi$. We show that $\psi^{\prime}$ is a valid function, a contradiction to the minimality of $\psi$.

Consider any $\bar{s} \in C_{S}(R)$ for some matrix $R$. We assume, without loss of generality, that $r^{1}, r^{2}$ and $r^{1}+r^{2}$ are columns of $R$ (otherwise, simply add the missing vectors as columns and put a value of 0 for the corresponding component of $\bar{s}$ ). Define $\tilde{s}$ as follows:

$$
\tilde{s}_{r}:= \begin{cases}\bar{s}+\bar{s}_{r^{1}+r^{2}} & \text { if } r=r^{1} \\ \bar{s}_{r}+\bar{s}_{r^{1}+r^{2}} & \text { if } r=r^{2} \\ 0 & \text { if } r=r^{1}+r^{2} \\ \bar{s}_{r} & \text { otherwise. }\end{cases}
$$

Note that $\tilde{s} \geq 0$ and $R \tilde{s}=R \bar{s} \in S$, thus $\tilde{s} \in C_{S}(R)$. Using the definitions of $\psi^{\prime}$ and $\tilde{s}$, it is easy to verify that $\sum \psi^{\prime}(r) \bar{s}_{r}=\sum \psi(r) \tilde{s}_{r} \geq 1$, where the last inequality follows from the facts that $\psi$ is valid and $\tilde{s} \in C_{S}(R)$. This shows that $\psi^{\prime}$ is valid.

We next show that $\psi$ is positively homogeneous. Suppose there exists $\tilde{r} \in \mathbb{R}^{n}$ and $\lambda>0$ such that $\psi(\lambda \tilde{r}) \neq \lambda \psi(\tilde{r})$. Without loss of generality we may assume that $\psi(\lambda \tilde{r})<\lambda \psi(\tilde{r})$. Define a function $\psi^{\prime}$ by $\psi^{\prime}(\tilde{r}):=\lambda^{-1} \psi(\lambda \tilde{r}), \psi^{\prime}(r):=\psi(r)$ for all $r \neq \tilde{r}$. It is easy to see that $\psi^{\prime}$ is valid, contradicting the fact that $\psi$ is minimal. Therefore $\psi$ is positively homogeneous.

(b) $\psi$ is nonnegative. Suppose $\psi(\tilde{r})<0$ for some $\tilde{r} \in \mathbb{Q}^{n}$. Let $q \in \mathbb{Z}_{+}$be such that $q \tilde{r} \in \mathbb{Z}^{n}$ and let $\bar{s} \in C_{S}(R)$, where $\tilde{r}$ is a column of $R$. Let $\tilde{s}$ be defined by $\tilde{s}_{\tilde{r}}:=\bar{s}_{\tilde{r}}+M q$ where $M$ is a positive integer, and $\tilde{s}_{r}:=\bar{s}_{r}$ for $r \neq \tilde{r}$. Then $\tilde{s} \in C_{S}(R)$ and $\sum \psi(r) \tilde{s}_{r}=\sum \psi(r) \bar{s}_{r}+\psi(\tilde{r}) M q$. Since $\psi(\tilde{r}) M q<0$ and $M$ is any positive integer, this sum can be made smaller than 1 , a contradiction to the validity of $\psi$.

Since $\psi$ is sublinear, $\psi$ is convex and therefore continuous. Thus, as $\psi$ is nonnegative over $\mathbb{Q}^{n}$ and $\mathbb{Q}^{n}$ is dense in $\mathbb{R}^{n}, \psi$ is nonnegative over $\mathbb{R}^{n}$. 
Let $K \subseteq \mathbb{R}^{n}$ be a closed convex set with the origin in its interior. A standard concept in convex analysis [61, 67] is that of gauge, which is the function $\gamma_{K}$ defined by

$$
\gamma_{K}(r):=\inf \left\{t>0: \frac{r}{t} \in K\right\} \quad \text { for all } r \in \mathbb{R}^{n} .
$$

Since the origin is in the interior of $K, \gamma_{K}(r)<+\infty$ for all $r \in \mathbb{R}^{n}$. Furthermore $\gamma_{K}(r) \leq 1$ if and only if $r \in K$, and $\operatorname{int}(K)=\left\{r \in \mathbb{R}^{n}: \gamma_{K}(r)<1\right\}$ : since $\gamma_{K}$ is a continuous function, $\gamma_{K}(\bar{r})<1$ implies $\gamma_{K}(r)<1$ for every $r$ close to $\bar{r}$, and since $\gamma_{K}$ is positively homogeneous, we have that $\gamma_{K}(\bar{r})=1$ implies $\gamma_{K}(r)>1$ if $r=(1+\varepsilon) \bar{r}$ for $\varepsilon>0$.

The following lemma is standard in convex analysis, see for instance [61].

Lemma 4.2. Given a closed convex set $K \subseteq \mathbb{R}^{n}$ with the origin in its interior, the gauge $\gamma_{K}$ is a nonnegative sublinear function.

Conversely, given a function $\gamma: \mathbb{R}^{n} \rightarrow \mathbb{R}$ which is nonnegative and sublinear, let

$$
K_{\gamma}:=\left\{x \in \mathbb{R}^{n}: \gamma(x) \leq 1\right\} .
$$

Then $K_{\gamma}$ is a closed convex set with the origin in its interior, and $\gamma$ is the gauge of $K_{\gamma}$.

Lemma 4.3. Assume $S=b+\mathbb{Z}^{n}$ for some $b \notin \mathbb{Z}^{n}$. Let $K \subseteq \mathbb{R}^{n}$ be a closed convex set with $0 \in \operatorname{int}(K)$ and let $\psi$ be the gauge of $K$. Then $\psi$ is a valid function for $S$ if and only if $K$ is $S$-free.

Proof. By Lemma 4.2, $\psi$ is sublinear. We prove the "if" part. Assume that $K$ is $S$-free and consider $s \in C_{S}(R)$ for some matrix $R$. That is, $\sum r s_{r}=b+x$, where $x \in \mathbb{Z}^{n}$ and the sum ranges over the columns $r$ of $R$. Then

$$
\sum \psi(r) s_{r}=\sum \psi\left(r s_{r}\right) \geq \psi\left(\sum r s_{r}\right)=\psi(b+x) \geq 1,
$$

where the first equality follows by positive homogeneity of $\psi$, the first inequality by subadditivity, and the last from the fact that $\psi$ is the gauge of $K$ and $b+x \notin \operatorname{int}(K)$ because $x \in \mathbb{Z}^{n}$ and $K$ is $S$-free.

For the "only if" part, assume $b+x \in \operatorname{int}(K)$, with $x \in \mathbb{Z}^{n}$. Let $R$ be the $n \times 1$ matrix $b+x$. Then the point defined by $s_{b+x}=1$ is in $C_{S}(R)$, and $\psi(b+x)<1$ because $\psi$ is the gauge of $K$ and $b+x \in \operatorname{int}(K)$. Thus $\psi$ is not a valid function for $S$.

The following theorem (see [17, 27]) shows the correspondence between minimal valid functions for $S$ and maximal $S$-free convex sets, when $S=b+\mathbb{Z}^{n}$.

TheOREM 4.4. Assume $S=b+\mathbb{Z}^{n}$ for some $b \notin \mathbb{Z}^{n}$. A function $\psi: \mathbb{R}^{n} \rightarrow \mathbb{R}$ is a minimal valid function for $S$ if and only if there exists some maximal $S$-free convex set $K \subseteq \mathbb{R}^{n}$ such that $0 \in \operatorname{int}(K)$ and $\psi$ is the gauge of $K$.

Proof. Assume that $\psi$ is a minimal valid function. By Lemma 4.1, $\psi$ is a nonnegative sublinear function, and by Lemma 4.2, $\psi$ is the gauge of a closed convex set $K$ such that $0 \in \operatorname{int}(K)$. Since $\psi$ is a valid function for $S$, by Lemma 4.3, $K$ is an $S$-free convex set. We prove that $K$ is a maximal $S$-free convex set. Suppose not, and let $K^{\prime}$ be an $S$-free convex set properly containing $K$. Let $\psi^{\prime}$ be the gauge of $K^{\prime}$. By Lemma 4.3, $\psi^{\prime}$ is a valid function, and since $K \subsetneq K^{\prime}$, we have that $\psi^{\prime} \leq \psi$ and $\psi^{\prime} \neq \psi$. This contradicts the minimality of $\psi$. The converse is straightforward. 
When $S=b+\mathbb{Z}^{n}$, in view of Theorem 4.4 characterizing minimal valid functions amounts to characterizing maximal $S$-free convex sets containing 0 in their interior. We will see that every maximal $S$-free convex set is a polyhedron. Therefore, if a maximal $S$-free convex set contains 0 in its interior, then it is a polyhedron of the form $K=\left\{x \in \mathbb{R}^{n}: a_{i} x \leq 1, i \in I\right\}$ for some finite set $I$. This turns out to be very useful, as it can be employed to obtain an explicit formula for the computation of the minimal valid function associated with $K$, i.e., the gauge of $K$. The formula is stated in the following theorem.

Theorem 4.5. Assume $S=b+\mathbb{Z}^{n}$ for some $b \notin \mathbb{Z}^{n}$. Then every maximal $S$-free convex set is a polyhedron. Moreover, if a maximal $S$-free polyhedron $K$ with $0 \in \operatorname{int}(K)$ is given by $K=\left\{x \in \mathbb{R}^{n}: a_{i} x \leq 1, \forall i \in I\right\}$ for some finite set $I$, then the gauge $\psi$ of $K$ is

$$
\psi(r)=\max _{i \in I} a_{i} r
$$

Since a set $K$ is a (maximal) $S$-free convex set if and only if $K-b$ is a (maximal) $\mathbb{Z}^{n}$-free convex set, the proof of Theorem 4.5 requires the characterization of maximal $\mathbb{Z}^{n}$-free convex sets and is postponed until the end of subsection 4.1.1.

\subsubsection{Maximal lattice-free convex sets}

We characterize the structure of maximal $\mathbb{Z}^{n}$-free sets in this section in Theorem 4.9 and then derive Theorem 4.5 as a consequence.

Our treatment uses basic facts about lattices. A lattice of dimension $t$ is a set of the type $\left\{x \in \mathbb{R}^{n}: x=\lambda_{1} a_{1}+\cdots+\lambda_{t} a_{t} ; \lambda_{1}, \ldots, \lambda_{t} \in \mathbb{Z}\right\}$, where $a_{1}, \ldots, a_{t}$ are linearly independent vectors in $\mathbb{R}^{n}$. It follows from this definition that $\mathbb{Z}^{n}$ is a lattice. We call a $\mathbb{Z}^{n}$-free convex set lattice-free. We refer to Chapter VII in the book of Barvinok [14] for an introduction to lattice theory.

A convex set $C$ is centrally symmetric with center $p$ if $x \in C$ implies $2 p-x \in C$. We will sometimes simply say $C$ is centrally symmetric, if there exists $p \in C$ such that $C$ is centrally symmetric with center $p$.

Theorem 4.6 (Minkowski's convex body theorem (see, e.g., [14])). Let $C \subseteq \mathbb{R}^{n}$ be a centrally symmetric convex set with center 0 . If $\operatorname{vol}(C)>2^{n}$, then $C$ contains a nonzero integer point. Moreover, if $C$ is compact, the condition can be relaxed to $\operatorname{vol}(C) \geq 2^{n}$.

A subspace $H \subseteq \mathbb{R}^{n}$ is a lattice subspace if $\operatorname{dim}\left(H \cap \mathbb{Z}^{n}\right)=\operatorname{dim}(H)$. That is, if $H$ can be generated by an integral basis. Equivalently, $H=\left\{x \in \mathbb{R}^{n}: A x=0\right\}$ for some $(n-\operatorname{dim}(H)) \times n$ integral matrix $A$ of full row-rank.

Given a linear subspace $L \subseteq \mathbb{R}^{n}$, there exists a unique minimal lattice subspace containing $L$. It is the intersection of all lattice subspaces containing $L$.

Lemma 4.7. Let $H \subseteq \mathbb{R}^{n}$ be a lattice subspace. Then $H+\mathbb{Z}^{n}$ is a closed set.

Proof. Since $H$ is a lattice subspace, by applying a suitable unimodular transformation we can assume that $H=\left\{x \in \mathbb{R}^{n}: x_{1}=\cdots=x_{k}=0\right\}$ for some $k \in\{0, \ldots, n\}$. Then $H+\mathbb{Z}^{n}=\left\{x \in \mathbb{R}^{n}: x_{1}, \ldots, x_{k} \in \mathbb{Z}\right\}$, which is a closed set. 
Proposition 4.8. Let $L \subseteq \mathbb{R}^{n}$ be a linear subspace and let $H$ be the minimal lattice subspace containing L. Then

$$
\operatorname{cl}\left(\operatorname{proj}_{L^{\perp}}\left(\mathbb{Z}^{n}\right)\right)=\left(H+\mathbb{Z}^{n}\right) \cap L^{\perp}=\left(H \cap L^{\perp}\right)+\Lambda
$$

for some lattice $\Lambda \subseteq L^{\perp}$ such that $\operatorname{dim}\left(H \cap L^{\perp}\right)+\operatorname{dim}(\Lambda)=\operatorname{dim}\left(L^{\perp}\right)$.

Proof. Note that $\operatorname{proj}_{L^{\perp}}\left(\mathbb{Z}^{n}\right)=\left(L+\mathbb{Z}^{n}\right) \cap L^{\perp}$. By Lemma 4.7, $\left(H+\mathbb{Z}^{n}\right) \cap L^{\perp}$ is a closed set containing $\left(L+\mathbb{Z}^{n}\right) \cap L^{\perp}$. It follows that $\operatorname{cl}\left(\operatorname{proj}_{L^{\perp}}\left(\mathbb{Z}^{n}\right)\right)=\operatorname{cl}\left(\left(L+\mathbb{Z}^{n}\right) \cap L^{\perp}\right) \subseteq\left(H+\mathbb{Z}^{n}\right) \cap L^{\perp}$.

To show the reverse inclusion, we first assume $H=\mathbb{R}^{n}$. In this case, $\left(H+\mathbb{Z}^{n}\right) \cap L^{\perp}=L^{\perp}$, thus we have to prove that for every $x \in L^{\perp}$ and $\varepsilon>0$, the ball $B(x, \varepsilon)$ intersects $\operatorname{proj}_{L^{\perp}}\left(\mathbb{Z}^{n}\right)$.

The proof is by (reverse) induction on $k:=\operatorname{dim}(L)$. The case $k=n$ is trivial. Now assume $k<n$. Fix $x \in L^{\perp}$ and $\varepsilon>0$ (with $\varepsilon<1$ without loss of generality), and assume by contradiction that no point in $\operatorname{proj}_{L^{\perp}}\left(\mathbb{Z}^{n}\right)$ belongs to $B(x, \varepsilon)$. We claim that $\operatorname{proj}_{L^{\perp}}\left(\mathbb{Z}^{n}\right)$ contains a nonzero vector $w$ such that $\|w\| \leq \varepsilon / 2$. To see this, define $\Lambda^{\prime}=L \cap \mathbb{Z}^{n}$. Since $L$ is not a lattice subspace (as $L \subsetneq H$ ), $\Lambda^{\prime}$ is a lattice of dimension smaller than $k$. Then there exists a nonzero vector $v \in L \cap\left\langle\Lambda^{\prime}\right\rangle^{\perp}$. Define $B^{\prime}=B(0, \varepsilon / 2) \cap\langle v\rangle^{\perp}$. Let $C$ be the centrally symmetric cylinder $C=B^{\prime}+[-\lambda v, \lambda v]$, where $\lambda>0$. For $\lambda$ large enough, $\operatorname{vol}(C)>2^{n}$, thus, by Minkowski's convex body theorem, $C$ contains a nonzero integer point $z$. Note that $z \notin L$ : otherwise, we would have $z \in C \cap L \cap \mathbb{Z}^{n}=C \cap \Lambda^{\prime} \subseteq B^{\prime}$; but $B^{\prime}$ contains no integer point other than the origin, as $\varepsilon<1$. Therefore $z \notin L$, which implies that its projection $w$ onto $L^{\perp}$ is not the origin. Note that $\|w\| \leq \varepsilon / 2$, as claimed.

We now claim that the unbounded cylinder $C^{\prime}=B(x, \varepsilon / 2)+\langle w\rangle$ contains no point from $\operatorname{proj}_{L^{\perp}}\left(\mathbb{Z}^{n}\right)$. Assume to the contrary that there exists $y \in \operatorname{proj}_{L^{\perp}}\left(\mathbb{Z}^{n}\right)$ such that $y=x^{\prime}+\mu w$, where $x^{\prime} \in B(x, \varepsilon / 2)$ and $\mu \in \mathbb{R}$. Then the point $y^{\prime}=y-\lfloor\mu\rfloor w=x^{\prime}+(\mu-\lfloor\mu\rfloor) w$ would also belong to $\operatorname{proj}_{L^{\perp}}\left(\mathbb{Z}^{n}\right)$; however

$$
\left\|y^{\prime}-x\right\|=\left\|y^{\prime}-x^{\prime}\right\|+\left\|x^{\prime}-x\right\| \leq\|w\|+\varepsilon / 2 \leq \varepsilon,
$$

thus $y^{\prime} \in B(x, \varepsilon)$, which is a contradiction, as $B(x, \varepsilon)$ contains no point from $\operatorname{proj}_{L^{\perp}}\left(\mathbb{Z}^{n}\right)$. Therefore $C^{\prime}$ contains no point from $\operatorname{proj}_{L^{\perp}}\left(\mathbb{Z}^{n}\right)$, as claimed.

Now, if $k=n-1$ (i.e., $L^{\perp}$ is a line), by choosing $\varepsilon$ arbitrarily small the norm of $w$ can be made arbitrarily small, and we conclude that $\operatorname{cl}\left(\operatorname{proj}_{L^{\perp}}\left(\mathbb{Z}^{n}\right)\right)=L^{\perp}$. So we assume $k<n-1$. Define $L^{\prime}=\langle L \cup\{w\}\rangle$. Since the minimal lattice subspace containing $L^{\prime}$ is $\mathbb{R}^{n}$, by induction $\operatorname{cl}\left(\operatorname{proj}_{\left(L^{\prime}\right) \perp}\left(\mathbb{Z}^{n}\right)\right)=\left(L^{\prime}\right)^{\perp}$. However, the projection of $C^{\prime}$ onto $\left(L^{\prime}\right)^{\perp}$ is a ball in $\left(L^{\prime}\right)^{\perp}$ that contains no point from $\operatorname{proj}_{\left(L^{\prime}\right) \perp}\left(\mathbb{Z}^{n}\right)$, a contradiction.

This concludes the proof for the case $H=\mathbb{R}^{n}$. If $H$ is subspace of dimension $n^{\prime}<n$, modulo a unimodular transformation we can assume that $H=\mathbb{R}^{n^{\prime}} \times\{0\}^{n-n^{\prime}}$. We can apply the result with respect to the ambient space $H$, which is equivalent to $\mathbb{R}^{n^{\prime}}$. We then have $\operatorname{cl}\left(\operatorname{proj}_{L^{\perp}}\left(H \cap \mathbb{Z}^{n}\right)=H \cap L^{\perp}\right.$. Similarly, for every $a \in \mathbb{Z}^{n}$ we have $\operatorname{cl}\left(\operatorname{proj}_{L^{\perp}}\left((H+a) \cap \mathbb{Z}^{n}\right)\right)=$ $(H+a) \cap L^{\perp}$. Then

$$
\operatorname{cl}\left(\operatorname{proj}_{L^{\perp}}\left(\mathbb{Z}^{n}\right)\right) \supseteq \bigcup_{a \in \mathbb{Z}^{n}} \operatorname{cl}\left(\operatorname{proj}_{L^{\perp}}\left((H+a) \cap \mathbb{Z}^{n}\right)\right)=\bigcup_{a \in \mathbb{Z}^{n}}(H+a) \cap L^{\perp}=\left(H+\mathbb{Z}^{n}\right) \cap L^{\perp} .
$$

It remains to show that $\left(H+\mathbb{Z}^{n}\right) \cap L^{\perp}=\left(H \cap L^{\perp}\right)+\Lambda$ for some lattice $\Lambda \subseteq L^{\perp}$ such that $\operatorname{dim}\left(H \cap L^{\perp}\right)+\operatorname{dim}(\Lambda)=\operatorname{dim}\left(L^{\perp}\right)$. Define $\Lambda=\operatorname{proj}_{H^{\perp}}\left(\mathbb{Z}^{n}\right)$. Since $H^{\perp}$ is a lattice subspace, 

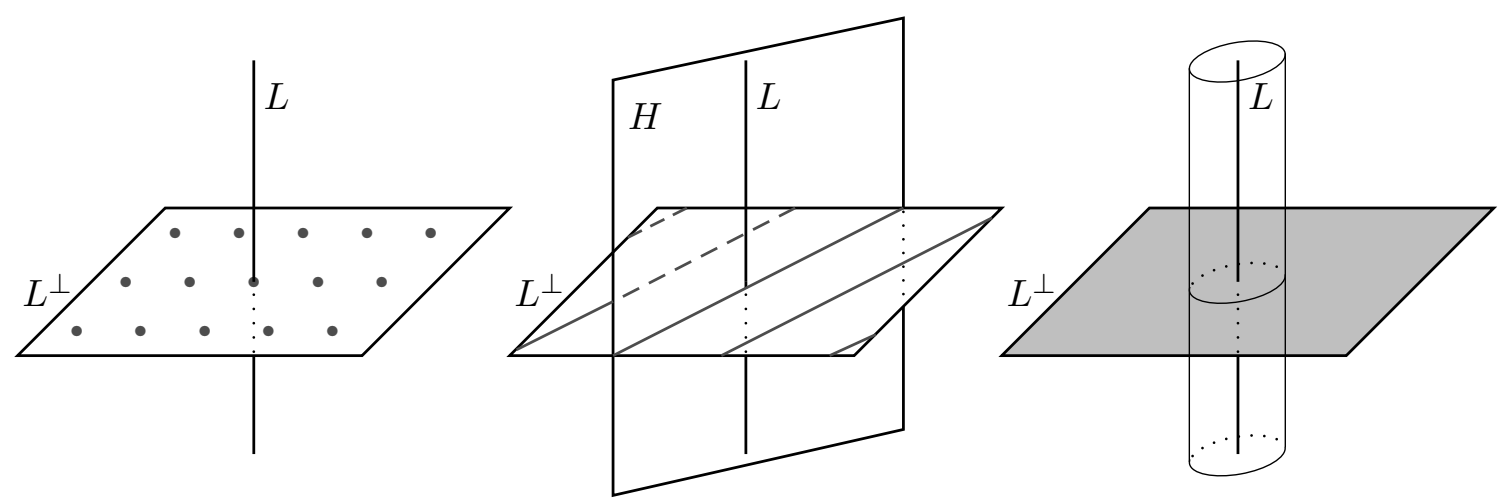

Figure 1: Illustration of Proposition 4.8 for $n=3$ with $L$ being a line. In the first picture, $H=L$ and the orthogonal projection of $\mathbb{Z}^{3}$ onto $L^{\perp}$ is a 2-dimensional lattice. In the second picture, $H$ is a plane, and the set $\operatorname{cl}\left(\operatorname{proj}_{L^{\perp}}\left(\mathbb{Z}^{3}\right)\right)$ is the union of discrete shifts of the line $H \cap L^{\perp}$. In the third picture, $H=\mathbb{R}^{3}$; the cylinder indicates that there are integer points in any neighborhood of $L$, and the projection of $\mathbb{Z}^{3}$ is dense in $L^{\perp}$ (i.e., its closure is $L^{\perp}$ ).

$\Lambda$ is a lattice. Moreover, since $L \subseteq H$, we have that $\Lambda \subseteq H^{\perp} \subseteq L^{\perp}$. By writing

$$
\left(H+\mathbb{Z}^{n}\right) \cap L^{\perp}=\operatorname{proj}_{H}\left(\left(H+\mathbb{Z}^{n}\right) \cap L^{\perp}\right)+\operatorname{proj}_{H^{\perp}}\left(\left(H+\mathbb{Z}^{n}\right) \cap L^{\perp}\right),
$$

and observing that $\operatorname{proj}_{H}\left(\left(H+\mathbb{Z}^{n}\right) \cap L^{\perp}\right)=\left(H \cap L^{\perp}\right)$ and $\operatorname{proj}_{H^{\perp}}\left(\left(H+\mathbb{Z}^{n}\right) \cap L^{\perp}\right)=\Lambda$, one concludes that $\left(H+\mathbb{Z}^{n}\right) \cap L^{\perp}=\left(H \cap L^{\perp}\right)+\Lambda$.

We illustrate the above proposition in the case $n=3$ with $L$ being a line. If $H=L$, then $\operatorname{cl}\left(\operatorname{proj}_{L^{\perp}}\left(\mathbb{Z}^{n}\right)\right)=\operatorname{proj}_{L^{\perp}}\left(\mathbb{Z}^{n}\right)$ is a lattice; if $\operatorname{dim}(H)=2$, then $\operatorname{cl}\left(\operatorname{proj}_{L^{\perp}}\left(\mathbb{Z}^{n}\right)\right)$ is the union of discrete shifts of $H \cap L^{\perp}$; finally if $H=\mathbb{R}^{3}, \operatorname{cl}\left(\operatorname{proj}_{L^{\perp}}\left(\mathbb{Z}^{n}\right)\right)=L^{\perp}$. See Figure 1.

Theorem 4.9. A set $K \subseteq \mathbb{R}^{n}$ is a maximal lattice-free convex set if and only if it satisfies one of the following conditions:

(a) $K=a+L$, where $a \in \mathbb{R}^{n}$ and $L$ is a subspace of dimension $n-1$ that is not a lattice subspace.

(b) $K$ is an $n$-dimensional polyhedron of the form $K=Q+L$, where $L$ is a lattice subspace of dimension $r$, with $r<n, Q$ is a polytope of dimension $n-r$, and the relative interior of every facet of $K$ contains an integer point.

Proof. We first prove the "if" direction. Assume that (a) holds; we show that $K$ is a maximal lattice-free convex set. Since the minimal lattice subspace containing $L$ is $\mathbb{R}^{n}$, Proposition 4.8 implies $\operatorname{cl}\left(\operatorname{proj}_{L^{\perp}}\left(\mathbb{Z}^{n}\right)\right)=L^{\perp}$. Then for every $\varepsilon>0$ and $a \in \mathbb{R}^{n}$, there is a point in $\mathbb{Z}^{n} \backslash(a+L)$ at distance at most $\varepsilon$ from $a+L$. Suppose that $K^{\prime}$ is a closed convex set that strictly contains $K$. Since $K^{\prime}$ is closed, it must contains a set of the form $[a, a+v]+L$ for some $v \in L^{\perp} \backslash\{0\}$. Then $\operatorname{int}\left(K^{\prime}\right)$ contains an integer point, a contradiction to the assumption that $K^{\prime}$ is latticefree. It follows that no lattice-free convex set strictly contains $K$ and thus $K$ is a maximal lattice-free convex set. 
Let now $K$ satisfy (b); we prove that $K$ is a maximal lattice-free convex set. If $K^{\prime}$ is a lattice-free convex set strictly containing $K$, then there is a facet $F$ of $K$ such that $\operatorname{relint}(F) \subseteq \operatorname{int}\left(K^{\prime}\right)$. Since relint $(F)$ contains an integer point, this point is in $\operatorname{int}\left(K^{\prime}\right)$, a contradiction to the assumption that $K^{\prime}$ is lattice-free. It follows that $K$ is a maximal lattice-free convex set.

We now prove the "only if" direction. We first assume that $K$ is a maximal lattice-free convex set with $\operatorname{dim}(K)<n$; we show that (a) holds. By maximality, $K$ is a hyperplane, hence $K=a+L$ for some $a \in \mathbb{R}^{n}$ and some linear subspace $L$ of dimension $n-1$. If $L$ is a lattice subspace, then $L=\left\{x \in \mathbb{R}^{n}: c x=0\right\}$ for some $c \in \mathbb{Z}^{n}$. Define $\alpha=c a$ and $K^{\prime}=\left\{x \in \mathbb{R}^{n}:\lfloor\alpha\rfloor \leq c x \leq\lfloor\alpha\rfloor+1\right\}$. Then $K^{\prime}$ is a lattice-free convex set that strictly contains $K$, a contradiction to the maximality of $K$. It follows that $L$ is not a lattice-subspace and (a) holds.

We finally show that if $K$ is an $n$-dimensional lattice-free convex set then (b) is satisfied. Claim. $\operatorname{lin}(K)=\operatorname{rec}(K)$.

Proof of Claim. We assume $\operatorname{rec}(K) \neq\{0\}$, otherwise the statement holds trivially. Define $K^{\prime}=K-\operatorname{rec}(K)$ and assume that there is an integer point $z \in \operatorname{int}\left(K^{\prime}\right)$. Choose $\varepsilon>0$ such that $B(z, \varepsilon) \subseteq \operatorname{int}\left(K^{\prime}\right)$ and let $v \in \operatorname{relint}(\operatorname{rec}(K))$. Then by Theorem 4.6 the set $B(z, \varepsilon)+\mathbb{R}_{+} v$ contains integer points arbitrarily far from $z$. On the other hand, by the choice of $v$, every point of the form $x+\lambda v$ with $x \in B(z, \varepsilon)$ and $\lambda$ large enough belongs to $\operatorname{int}(K)$. This contradicts the fact that $K$ is lattice-free. Therefore $\operatorname{int}\left(K^{\prime}\right)$ contains no integer point. Since $K$ is maximally lattice-free and $K \subseteq K^{\prime}$, it follows that $K=K^{\prime}$, i.e., $\operatorname{lin}(K)=\operatorname{rec}(K)$. $\diamond$

Claim. $\operatorname{lin}(K)$ is a lattice subspace.

Proof of Claim. Define $L=\operatorname{lin}(K)$ and let $H$ be the minimal lattice subspace containing $L$. If $L$ is not a lattice subspace, then $L \subsetneq H$, and therefore there exists a nonzero vector $v \in H \cap L^{\perp}$. Define $K^{\prime}=K+\langle v\rangle$ and assume that $\operatorname{int}\left(K^{\prime}\right)$ contains an integer point $z$. Then $z=x+\lambda v$ for some $x \in \operatorname{int}(K)$ and $\lambda \in \mathbb{R}$. Since $x+L \subseteq H+\mathbb{Z}^{n}$, by Proposition 4.8 we have $(x+L) \cap L^{\perp} \subseteq \operatorname{cl}\left(\operatorname{proj}_{L^{\perp}}\left(\mathbb{Z}^{n}\right)\right)$. This implies that there are integer points that are arbitrarily close to $x+L$. Since $x+L \subseteq \operatorname{int}(K)$, this contradicts the fact that $K$ is lattice-free. We conclude that $K^{\prime}$ is lattice-free, which is a contradiction to the maximality of $K$.

By the claims, $K=Q+L$ where $L$ is a lattice subspace and $Q=K \cap L^{\perp}=\operatorname{proj}_{L^{\perp}}(K)$. Note that $Q$ is a bounded set, $0 \leq \operatorname{dim}(L) \leq n-1$ and $\operatorname{dim}(Q)=n-\operatorname{dim}(L)$.

It remains to prove that $Q$ is a polytope and the relative interior of every facet of $K$ contains an integer point. Since $L$ is a lattice subspace, by Proposition 4.8 , $\operatorname{proj}_{L^{\perp}}\left(\mathbb{Z}^{n}\right)$ is a lattice $\Lambda$. Since $K$ is a maximal lattice-free convex set and $K=Q+L$, then $\operatorname{int}(Q) \cap \Lambda=\emptyset$ and $Q$ is a maximal $\Lambda$-free convex set (in the space $L^{\perp}$ ). Since $Q \subseteq L^{\perp}$ is a bounded set, then $Q \subseteq B \subseteq L^{\perp}$, where $B$ is a box. Let $B \cap \Lambda=\left\{z_{1}, \ldots, z_{k}\right\}$. Since $\operatorname{int}(Q) \cap \Lambda=\emptyset$, for every $z_{i}$ there exists an half-space $H_{i}$ containing $Q$ and having $z_{i}$ on the boundary. Then $Q \subseteq B \cap H_{1}, \cdots \cap H_{k}$, and in fact $Q=B \cap H_{1}, \cdots \cap H_{k}$ by maximality of $Q$. This shows that $Q$ is a polytope.

Assume that a facet $F$ of $Q$ is such that $\operatorname{relint}(F) \cap \Lambda=\emptyset$. (Equivalently, the relative interior of the facet $F+L$ of $K$ does not contain an integer point.) Since $F$ is a polytope and $\Lambda$ is a lattice, every point in $\Lambda$ is at distance at least $\varepsilon$ from $\operatorname{relint}(F)$, for some $\varepsilon>0$. Let 
$Q^{\prime}$ be obtained from $Q$ by relaxing $F$ by $\varepsilon$. By construction, $\operatorname{relint}\left(Q^{\prime}\right) \cap \Lambda=\emptyset$ and $Q^{\prime} \supsetneq Q$. Let $K^{\prime}=Q^{\prime}+L$. Then $K^{\prime}$ is a lattice-free convex set and $K^{\prime} \supsetneq K$, a contradiction to the maximality of $K$.

We remark that the above theorem also holds for maximal $\left(b+\mathbb{Z}^{n}\right)$-free convex sets, except that the last part of condition (b) becomes: "the relative interior of every facet of $K$ contains a point in $b+\mathbb{Z}^{n \prime \prime}$.

The original proof of Theorem 4.9 of Basu et al. [17] uses the Dirichlet approximation theorem. The subsequent (short) proof of Averkov [8] uses the convex body theorem of Minkowski (Theorem 4.6) which implies the theorem of Dirichlet.

As shown in Theorem 4.9, maximal lattice-free convex sets are polyhedra. The following result bounds the number of facets of these polyhedra.

Theorem 4.10 (Doignon [45], Bell [26], Scarf [68]). Let $K \subseteq \mathbb{R}^{n}$ be a maximal lattice-free convex set. Then $K$ is a polyhedron with at most $2^{n}$ facets.

Proof. By Theorem 4.9, every facet of $K$ contains a point in $\mathbb{Z}^{n}$ in its relative interior. If $K$ has more than $2^{n}$ facets, then $K$ has two facets whose relative interiors contain points say $z_{1}, z_{2} \in \mathbb{Z}^{n}$ that are congruent modulo 2 , i.e., $z_{1}-z_{2}$ has even components. But then $z=\frac{1}{2} z_{1}+\frac{1}{2} z_{2}$ is an integral point in $\operatorname{int}(K)$, a contradiction.

We can now prove the correctness of formula (4.1) to compute the gauge $\psi$ of $K$.

Proof of Theorem 4.5. Recall that $K=\left\{x \in \mathbb{R}^{n}: a_{i} x \leq 1, i \in I\right\}$ is a maximal $\left(b+\mathbb{Z}^{n}\right)$-free convex set with $0 \in \operatorname{int}(K)$. Then $\operatorname{dim}(K)=n$ and $K$ satisfies (b) of Theorem 4.9, hence $\operatorname{dim}(\operatorname{rec}(K))<n$. Since $\operatorname{rec}(K)=\left\{x \in \mathbb{R}^{n}: a_{i} x \leq 0, i \in I\right\}$ and $0 \in K$, then $\operatorname{rec}(K) \subseteq K$.

Fix $r \in \mathbb{R}^{n}$ and let $k$ be an index in $I$ such that $a_{k} r=\max _{i \in I} a_{i} r$. Assume that $a_{k} r<0$. Then there exists $\varepsilon>0$ such that $a_{k} r^{\prime}<0$ for every $r^{\prime} \in B(r, \varepsilon)$, and therefore $\operatorname{dim}(\operatorname{rec}(K))=$ $n$, a contradiction.

It follows that $a_{k} r \geq 0$. If $a_{k} r=0$ then $r$ belongs to $\operatorname{rec}(K)$. In this case $\psi(r)=0$ and (4.1) holds. So we assume $a_{k} r>0$. Define $t=a_{k} r$. Then, for $i \in I, a_{i}(r / t) \leq 1$, and thus $\psi(r) \leq t$. On the other hand, if $t^{\prime}<t$ then $a_{k}\left(r / t^{\prime}\right)>1$. This proves that $\psi(r)=t$.

\subsection{General sets $S$}

In this section we show how the theory developed in Section 4.1 for the case $S=b+\mathbb{Z}^{n}$ extends to more general closed sets $S \subseteq \mathbb{R}^{n} \backslash\{0\}$.

\subsubsection{Minimal valid functions}

Conforti, Cornuéjols, Daniilidis, Lemaréchal and Malick [31] studied the link between minimal valid functions and $S$-free convex sets, independently from the structure of maximal $S$-free convex sets. We summarize some of their results here.

Theorem 4.11. Given a closed set $S \subseteq \mathbb{R}^{n} \backslash\{0\}$, let $\psi: \mathbb{R}^{n} \rightarrow \mathbb{R}$ be a valid function for $S$, and let $\psi^{\prime}$ be defined as

$$
\psi^{\prime}(\hat{r})=\inf \left\{\sum \psi(r) s_{r}: \sum r s_{r}=\hat{r}, s_{r} \geq 0\right\} \text { for every } \hat{r} \in \mathbb{R}^{n} .
$$

Then $\psi^{\prime}$ is a valid function $\mathbb{R}^{n} \rightarrow \mathbb{R}$ which is sublinear. 
In the above theorem, the summations are taken over all finite subsets of $\mathbb{R}^{n}$. We also stress that the above theorem implies that $\psi^{\prime}$ cannot take the value $-\infty$. If $\psi$ and $\psi^{\prime}$ are as in Theorem 4.11, then $\psi^{\prime} \leq \psi$ by definition. Therefore to characterize minimal valid functions, one can concentrate on sublinear functions. However, unlike the case $S=b+\mathbb{Z}^{n}$ (see Lemma 4.1), a minimal valid function can take negative values.

Given a sublinear function $\rho$, let

$$
V_{\rho}:=\left\{r \in \mathbb{R}^{n}: \rho(r) \leqslant 1\right\} .
$$

Then $V_{\rho}$ is a closed convex set and $0 \in \operatorname{int}\left(V_{\rho}\right)$. Conversely, given a closed convex set $V$ with $0 \in \operatorname{int}(V)$, a sublinear function $\rho$ such that $V=V_{\rho}$ is a representation of $V$.

TheOrem 4.12. Let $S \subseteq \mathbb{R}^{n} \backslash\{0\}$ be a closed set, let $\rho$ be a sublinear function, and let $V_{\rho}$ be defined as in (4.2). Then $\rho$ is a valid function for $S$ if and only $V_{\rho}$ is $S$-free.

In view of Theorems 4.11 and 4.12, to characterize minimal valid functions for $S$ one has to study representations of $S$-free convex sets, which are in general not unique. However, these representations satisfy the following:

TheOREM 4.13. Let $V \subseteq \mathbb{R}^{n}$ be a closed convex set with $0 \in \operatorname{int}(V)$ and let $\rho$ be a representation of $V$. Then

$$
\rho(r) \leq 0 \Longleftrightarrow r \in \operatorname{rec}(V) \text {, and } \rho(r)<0 \Longrightarrow r \in \operatorname{int}(\operatorname{rec}(V)) .
$$

Furthermore all representations of $V$ coincide in $V \backslash \operatorname{int}(\operatorname{rec}(V))$.

Theorem 4.9 shows that if a polyhedron $K=\left\{x \in \mathbb{R}^{n}: a_{i} x \leq 1, i \in I\right\}$ with $0 \in \operatorname{int}(K)$ is a maximal $\left(b+\mathbb{Z}^{n}\right)$-free convex set, then $\operatorname{dim}(\operatorname{rec}(K))<n$, hence $\operatorname{int}(\operatorname{rec}(K))=\emptyset$. Therefore, by Theorem 4.13, the gauge of $K$ is the unique representation of $K$.

However, even in the case $S=\mathbb{Z}_{+}^{n}$, uniqueness does not hold. Basu, Conforti, Cornuéjols and Zambelli [18] show that in this case a maximal $S$-free convex set is always a polyhedron $K$, but in general $K$ admits an infinite set of representations; see also [31.

As an example, consider the polyhedron $K=\left\{r \in \mathbb{R}^{2}: r_{1} \leq 1, r_{2} \leq 1, \frac{1}{2}\left(-r_{1}+r_{2}\right) \leq 1\right\}$. Then the function $\gamma_{K}$ defined as $\gamma_{K}(r)=\max \left\{0, r_{1}, r_{2}, \frac{1}{2}\left(-r_{1}+r_{2}\right)\right\}$ is a representation of $K$ and indeed $\gamma_{K}$ is the gauge of $K$. However, the function $\mu_{K}$ defined as $\mu_{K}(r)=$ $\max \left\{r_{1}, r_{2}, \frac{1}{2}\left(-r_{1}+r_{2}\right)\right\}$ is also a representation of $K$, and we will see that $\mu_{K}$ is the smallest representation of $K$, in the sense that $\mu_{K} \leq \rho_{K}$ for every representation $\rho_{K}$ of $K$.

The polar of a set $V \subseteq \mathbb{R}^{n}$ is the set $V^{\circ}=\left\{r \in \mathbb{R}^{n}: r d \leq 1\right.$ for all $\left.d \in V\right\}$. A set $G \subseteq \mathbb{R}^{n}$ is a prepolar of $V$ if $G^{\circ}=V$. If $V$ is a closed convex set and $0 \in \operatorname{int}(V)$, then $V^{\circ}$ is a bounded set and $\left(V^{\circ}\right)^{\circ}=V$. Therefore in this case the polar of $V$ is itself a prepolar, but $V$ may have other prepolars.

For the polyhedron $K$ given in the above example, $K^{\circ}=\operatorname{conv}\left\{0, r_{1}, r_{2}, \frac{1}{2}\left(-r_{1}+r_{2}\right)\right\}$ and $\left(K^{\circ}\right)^{\circ}=K$, so $K^{\circ}$ is a prepolar of $K$. However, let $G=\operatorname{conv}\left\{r_{1}, r_{2}, \frac{1}{2}\left(-r_{1}+r_{2}\right)\right\}$. Then $G^{\circ}=K$, so $G$ is also a prepolar of $K$.

The support function of a set $G \subset \mathbb{R}^{n}$ is

$$
\sigma_{G}(r):=\sup _{d \in G} d r .
$$


The support function is sublinear and remains unchanged if $G$ is replaced by its closed convex hull: $\sigma_{G}=\sigma_{\overline{\operatorname{conv}}(G)}$. Conversely, any sublinear function $\sigma$ is the support function of a closed convex set, defined by

$$
G_{\sigma}:=\left\{d \in \mathbb{R}^{n}: d r \leqslant \sigma(r) \text { for all } r \in \mathbb{R}^{n}\right\} .
$$

THEOREM 4.14. Let $V$ be a closed convex set with $0 \in \operatorname{int}(V)$. Then $V$ admits an inclusionwise smallest prepolar. The smallest representation of $V$ is the support function of the smallest prepolar of $V$.

If $V$ is a polyhedron $K$ and $0 \in \operatorname{int}(K)$, the support function of the smallest prepolar of $V$ can be computed with formula (4.1).

TheOREM 4.15. Let $a_{i} x \leq 1, i \in I$ be an irredundant representation of a polyhedron $K$ with $0 \in \operatorname{int}(K)$. Then $\left\{a_{i}, i \in I\right\}$ is the smallest prepolar of $K$. Hence the smallest representation of $K$ is the function $\mu_{K}$ defined as

$$
\mu_{K}(r)=\max _{i \in I} a_{i} r
$$

For more details on the theory of smallest representations, see Basu, Cornuéjols and Zambelli [20] and Conforti et al. [31].

\subsubsection{Maximal $S$-free convex sets}

Theorem 4.10 extends to more general sets $S$. Specifically, when $S=\left(b+\mathbb{Z}^{n}\right) \cap Q$, where $Q$ is a rational polyhedron, the authors in [18 prove that every maximal $S$-free convex set is a polyhedron with at most $2^{n}$ facets. Morán and Dey [65] showed that the same statement holds when $S=\left(b+\mathbb{Z}^{n}\right) \cap C$, where $C$ is a convex set. We illustrate below how this result can be obtained by means of a connection with the Helly number studied by Averkov [7].

For a set $S \subseteq \mathbb{R}^{n}$, Averkov introduces the following definitions and parameters.

Definition $4.16(f(S)$, largest number of facets in a $n$-dimensional maximal $S$-free convex set). If every $n$-dimensional maximal $S$-free convex set is a polyhedron with at most $k$ facets, $f(S)$ is the minimal $k$ as above. If there exist no $n$-dimensional maximal $S$-free convex sets (e.g., for $S=\mathbb{R}^{n}$ ), define $f(S)=-\infty$. If there exist maximal $S$-free convex sets which are not polyhedra or maximal $S$-free polyhedra with an arbitrarily large number of facets, define $f(S)=+\infty$.

A set $A \subseteq \mathbb{R}^{n}$ is called $S$-convex if it is of the form $A=S \cap C$ for some convex set $C \subseteq \mathbb{R}^{n}$.

DeFINITION 4.17 (Helly number). Given a nonempty family $\mathcal{F}$ of sets, the Helly number $h(\mathcal{F})$ of $\mathcal{F}$ is defined as follows. If $\mathcal{F}=\emptyset, h(\mathcal{F})=0$. If $\mathcal{F} \neq \emptyset$ and there exists $k$ such that

$$
F_{1} \cap \cdots \cap F_{m}=\emptyset \Longrightarrow \exists i_{1}, \ldots, i_{\ell} \in[m], \ell \leq k, \text { such that } F_{i_{1}} \cap \cdots \cap F_{i_{\ell}}=\emptyset
$$

for all $F_{1} \ldots, F_{m} \in \mathcal{F}$, then $h(\mathcal{F})$ is the minimal $k$ as above. In all other cases, $h(\mathcal{F})=+\infty$. 
For $S \subseteq \mathbb{R}^{n}$ we use the notation $h(S)=h\left(\left\{S \cap C: C \subseteq \mathbb{R}^{n}\right.\right.$ is convex $\left.\}\right)$. That is, $h(S)$ is the Helly number of the family of all $S$-convex sets. Note that if $C$ is a convex set, then $h(S \cap C) \leq h(S)$. When $S=\mathbb{Z}^{n}$, Doignon [45] proves $f(S)=h(S)=2^{n}$ - see Theorems 4.10] and 4.20 ,

Averkov [7] proves the following.

TheOrem 4.18. Given $S \subseteq \mathbb{R}^{n}, f(S) \leq h(S)$.

Proof. We prove the theorem under the assumption that every maximal $S$-free convex set is a polyhedron. Let $K \subseteq \mathbb{R}^{n}$ be a maximal $S$-free polyhedron. Represent $K$ as the intersection of closed half-spaces $H_{1}, \ldots, H_{m}$. Then $S \cap \operatorname{int}\left(H_{1}\right) \cap \cdots \cap \operatorname{int}\left(H_{m}\right)=\emptyset$. By the definition of Helly number, there exist indices $i_{1}, \ldots, i_{\ell} \in[m]$, with $\ell \leq h(S)$, such that $S \cap \operatorname{int}\left(H_{i_{1}}\right) \cap \cdots \cap$ $\operatorname{int}\left(H_{i_{\ell}}\right)=\emptyset$. It follows that $K \subseteq K^{\prime}:=H_{i_{1}} \cap \cdots \cap H_{i_{\ell}}$, where $K^{\prime}$ is an $S$-free polyhedron with at most $h(S)$ facets. By maximality of $K$, we have $K=K^{\prime}$ and thus $K$ has at most $h(S)$ facets.

In the more general case in which maximal $S$-free convex sets are not guaranteed to be polyhedra, Averkov [7] derives inequality $f(S) \leq h(S)$ by approximating a maximal $S$-free convex set $K$ with a sequence of polyhedra $K_{t} \subseteq K$ that converges to $K$.

Averkov [7] proves that if $S$ is a discrete set (i.e., $S \cap B$ is finite for every bounded set $\left.B \subseteq \mathbb{R}^{n}\right)$, then $h(S)=f(S)$. We sketch his proof below.

THEOREM 4.19. If $S \subseteq \mathbb{R}^{n}$ is discrete, then $h(S)=f(S)$.

Proof. For the sake of simplicity, we assume that $S$ is finite; Averkov [7] then shows how to derive the result for discrete sets $S$ by means of limit arguments.

Since $S$ is finite, every $S$-convex set can be written as the intersection of $S$ with finitelymany open half-spaces. By using this, one easily verifies that $h(S)=h(\mathcal{F})$, where $\mathcal{F}$ is the family of all sets of the form $S \cap H$, with $H$ being an open half-space. Thus it is enough to prove that $h(\mathcal{F}) \leq f(S)$.

Let $H_{1}, \ldots, H_{m}$ be open half-spaces such that $H_{1} \cap \cdots \cap H_{m} \cap S=\emptyset$. We need to show that there exists $I \subseteq\{1, \ldots, m\}$ with $|I| \leq f(S)$ such that $\bigcap_{i \in I} H_{i} \cap S=\emptyset$. For $i=1, \ldots, m$, $H_{i}=\left\{x \in \mathbb{R}^{n}: a_{i} x<\beta_{i}\right\}$ for some $a_{i} \in \mathbb{R}^{n}$ and $\beta_{i} \in \mathbb{R}$.

For $i=1, \ldots, n$ we do the following. Define $\gamma_{i}$ as the supremum of the values $\gamma$ such that, if $H_{i}$ is replaced with $\left\{x \in \mathbb{R}^{n}: a_{i} x<\gamma\right\}$, then we still have empty intersection with $S$. Let $I$ be the set of indices for which $\gamma_{i}<+\infty$. For $i \in I$, redefine $H_{i}:=\left\{x \in \mathbb{R}^{n}: a_{i} x<\gamma_{i}\right\}$. One verifies that $\bigcap_{i \in I} H_{i} \cap S=\emptyset$.

By definition of $\gamma_{i}$ and by the finiteness of $S$, one can check that every inequality $a_{i} x \leq$ $\gamma_{i}, i \in I$ defines a facet of the polyhedron $K=\left\{x \in \mathbb{R}^{n}: a_{i} x \leq \gamma_{i}, i \in I\right\}$, and every such facet contains an integer point in its relative interior. Then $K$ is a maximal $S$-free convex set and thus has at most $f(S)$ facets. In other words, $|I| \leq f(S)$, and thus $h(\mathcal{F}) \leq f(S)$.

THEOREM 4.20. $h\left(b+\mathbb{Z}^{n}\right)=2^{n}$ for every $b \in \mathbb{R}^{n}$.

Proof. It suffices to consider the case $b=0$. By Theorem 4.10, $f\left(\mathbb{Z}^{n}\right) \leq 2^{n}$. Since there exist maximal lattice-free polyhedra with $2^{n}$ facets, $f\left(\mathbb{Z}^{n}\right)=2^{n}$. Then Theorem 4.19 yields $h\left(\mathbb{Z}^{n}\right)=f\left(\mathbb{Z}^{n}\right)=2^{n}$. 
We can now prove the result by Dey and Morán 65.

Theorem 4.21. Let $S=\left(b+\mathbb{Z}^{n}\right) \cap C$, where $b \in \mathbb{R}^{n}$ and $C$ is a convex set. Then $f(S) \leq 2^{n}$.

Proof. Recall that $h(S) \leq h\left(b+\mathbb{Z}^{n}\right)$, as $S=\left(b+\mathbb{Z}^{n}\right) \cap C$ and $C$ is a convex set. Then, by Theorems 4.19 and 4.20, $f(S)=h(S) \leq h\left(b+\mathbb{Z}^{n}\right)=2^{n}$.

Averkov also shows an extension of this result, namely $f(S) \leq 2^{n}$ for every $\left(\mathbb{Z}^{n} \times \mathbb{R}^{p}\right)$ convex set $S$ [7]. More recently, Aliev, Bassett, De Loera, Louveaux generalize Theorem 4.10 in the following way 3]. Given natural numbers $n, k$, they prove the existence of a constant $c(n, k)$ (depending only on $n, k)$ such that any maximal polyhedron with exactly $k$ integer points in its interior has at most $c(n, k)$ facets.

We mention that for $S=b+\mathbb{Z}^{2}$, a complete classification of maximal $S$-free convex sets in $\mathbb{R}^{2}$ has been obtained in [44] by Dey and Wolsey; Cornuéjols and Margot [36] give an alternate proof. The classification states that any maximal $\left(b+\mathbb{Z}^{2}\right)$-free convex set is one of five different types:

1. A split, i.e., the intersection of two half spaces whose corresponding hyperplanes are parallel and contain infinitely many points from $S$.

2. A type 1 triangle, which is an affine unimodular transformation of $\operatorname{conv}\left\{0,2 e^{1}, 2 e^{2}\right\}$.

3. A type 2 triangle, which has a single side with multiple points from $S$ in its relative interior, and the other two sides have exactly one point from $S$ in their relative interior. Moreover, the line passing through these two points is parallel to the third side.

4. A type 3 triangle, which contains exactly three points from $S$ on its boundary, one in the relative interior of each side.

5. A quadrilateral where each side has exactly one point from $S$ in its relative interior, and the four points form the translation of a fundamental parallelepiped of $\mathbb{Z}^{2}$.

The classification is also completely known in $\mathbb{R}^{2}$ when $S=\left(b+\mathbb{Z}^{2}\right) \cap Q$, where $Q$ is a rational polyhedron [25]. A partial classification for $\mathbb{R}^{3}$ when $S=b+\mathbb{Z}^{3}$ has been obtained in [10], which provides a complete description of all the $S$-free tetrahedra with integral vertices (which extend the type 1 triangles in $\mathbb{R}^{2}$ ). However, a complete classification for $\mathbb{R}^{3}$ has not been obtained.

When $S=\left(b+\mathbb{Z}^{n}\right) \cap Q$, where $Q$ is a rational polyhedron, Basu et al. 18, prove the following sharpening of Theorem 4.21 ,

THEOREM 4.22. Let $S=\left(b+\mathbb{Z}^{n}\right) \cap Q$, where $Q$ is a rational polyhedron such that $\operatorname{dim}(\operatorname{conv}(S))=$ $n$. A set $K \subseteq \mathbb{R}^{n}$ is a maximal $S$-free convex set if and only if one of the following holds:

(i) $K$ is a polyhedron such that $K \cap \operatorname{conv}(S)$ has nonempty interior, $K$ does not contain any point of $S$ in its interior and there is a point of $S$ in the relative interior of every facet of $K$. The cone $\operatorname{rec}(K \cap \operatorname{conv}(S))$ is rational and it is contained in $\operatorname{lin}(K)$.

(ii) $K$ is a half-space of $\mathbb{R}^{n}$ such that $K \cap \operatorname{conv}(S)$ has empty interior and the boundary of $K$ is a supporting hyperplane of $\operatorname{conv}(S)$.

(iii) $K$ is a hyperplane of $\mathbb{R}^{n}$ such that $\operatorname{lin}(K) \cap \operatorname{rec}(\operatorname{conv}(S))$ is not a rational polyhedron. 


\section{The pure integer model}

In this section, we consider the pure integer model (2.4), which we rewrite here for convenience:

$$
I_{S}(P):=\left\{y \in \mathbb{Z}_{+}^{\ell}: P y \in S\right\} .
$$

We assume throughout this section that $S=b+\mathbb{Z}^{n}$ for some $b \in \mathbb{R}^{n} \backslash \mathbb{Z}^{n}$. This case was introduced and studied by Gomory and Johnson [52, 53]. In the literature, this model is frequently refereed to under the name of infinite group problem. Recently, Yildiz and Cornuéjols extend the analysis to more general $S$ [70, but we will not cover their work in this survey.

Although for $S=b+\mathbb{Z}^{n}$ there do exist integer valid functions that take negative values, we only concentrate on nonnegative integer valid functions in this survey. Some justification for the nonnegativity assumption can be given as follows. If $P$ is a rational matrix and $I_{S}(P) \neq \emptyset$, then $\operatorname{rec}\left(\operatorname{conv}\left(I_{S}(P)\right)\right)=\mathbb{R}_{+}^{\ell}$ 34]. Therefore every inequality that is essential for a linear description of $\operatorname{conv}\left(I_{S}(P)\right)$ has nonnegative coefficients.

\subsection{Minimal integer valid functions}

Recall that an integer valid function $\pi$ for $S$ is said to be minimal if there is no integer valid function $\pi^{\prime} \neq \pi$ such that $\pi^{\prime}(p) \leq \pi(p)$ for all $p \in \mathbb{R}^{n}$. Notice that if $\pi$ is a nonnegative integer valid function which is minimal, then $\pi \leq 1$. Minimal integer valid functions for $S$ were characterized by Gomory and Johnson [52.

Recall that a function $\pi: \mathbb{R}^{n} \rightarrow \mathbb{R}$ is subadditive if $\pi\left(p^{1}+p^{2}\right) \leq \pi\left(p^{1}\right)+\pi\left(p^{2}\right)$ for all $p^{1}, p^{2} \in$ $\mathbb{R}^{n}$. When $S=b+\mathbb{Z}^{n}$, we say that $\pi$ satisfies the symmetry condition if $\pi(p)+\pi(b-p)=1$ for all $p \in \mathbb{R}^{n}$. Finally, $\pi$ is periodic modulo $\mathbb{Z}^{n}$ if $\pi(p)=\pi(p+w)$ for all $w \in \mathbb{Z}^{n}$.

Theorem 5.1 (Gomory and Johnson [52]). Let $S=b+\mathbb{Z}^{n}$ for some $b \notin \mathbb{Z}^{n}$, and let $\pi: \mathbb{R}^{n} \rightarrow \mathbb{R}$ be a nonnegative function. Then $\pi$ is a minimal integer valid function for $S$ if and only if $\pi(w)=0$ for all $w \in \mathbb{Z}^{n}, \pi$ is subadditive, and $\pi$ satisfies the symmetry condition. (These conditions imply that $\pi$ is periodic modulo $\mathbb{Z}^{n}$ and $\pi(b+w)=1$ for every $w \in \mathbb{Z}^{n}$.)

Proof. We first prove the "only if" part of the statement. Assume that $\pi$ is a minimal integer valid function for $S$. We need to show the following three facts.

(a) $\pi(w)=0$ for every $w \in \mathbb{Z}^{n}$. Define by $\pi^{\prime}(p)=\pi(p)$ if $p \notin \mathbb{Z}^{n}$ and $\pi^{\prime}(w)=0$ for all $w \in \mathbb{Z}^{n}$. If $\bar{y} \in \mathbb{Z}_{+}^{\ell}$ is a point in $I_{S}(P)$ for some $P$, then so is $\tilde{y}$ defined by $\tilde{y}_{p}=\bar{y}_{p}$ if $p \notin \mathbb{Z}^{n}$, and $\tilde{y}_{p}=0$ if $p \in \mathbb{Z}^{n}$. Moreover, $\sum \pi^{\prime}(p) \bar{y}_{p}=\sum \pi(p) \tilde{y}_{p} \geq 1$ since $\pi$ is valid. Therefore, $\pi^{\prime}$ is valid. Minimality of $\pi$ implies $\pi^{\prime}=\pi$.

(b) $\pi$ is subadditive. Let $p^{1}, p^{2} \in \mathbb{R}^{n}$. We need to show $\pi\left(p^{1}\right)+\pi\left(p^{2}\right) \geq \pi\left(p^{1}+p^{2}\right)$. This inequality holds when $p^{1}=0$ or $p^{2}=0$ because $\pi(0)=0$. Assume now that $p^{1} \neq 0, p^{2} \neq 0$ and $\pi\left(p^{1}\right)+\pi\left(p^{2}\right)<\pi\left(p^{1}+p^{2}\right)$. Define the function $\pi^{\prime}$ as follows: $\pi^{\prime}\left(p^{1}+p^{2}\right)=\pi\left(p^{1}\right)+\pi\left(p^{2}\right)$ and $\pi^{\prime}(p)=\pi(p)$ for $p \neq p^{1}+p^{2}$. The same argument used in Lemma 4.1 shows that $\pi^{\prime}$ is valid.

Now (a) and (b) imply that $\pi$ is periodic. This is because for any $p \in \mathbb{R}^{n}$ and $w \in \mathbb{Z}^{n}$, $\pi(p+w) \leq \pi(p)+\pi(w)=\pi(p)$ where the inequality is from (b) and the equality is from (a). Similarly, $\pi(p+w)=\pi(p+w)+\pi(-w) \geq \pi(p+w-w)=\pi(p)$. 
(c) $\pi$ satisfies the symmetry condition. Suppose there exists $\tilde{p} \in \mathbb{R}^{n}$ such that $\pi(\tilde{p})+\pi(b-\tilde{p}) \neq$ 1. Since $\pi$ is valid, $\pi(\tilde{p})+\pi(b-\tilde{p})=1+\delta$ where $\delta>0$. Since $\pi(p) \leq 1$ for all $p \in \mathbb{R}^{n}$ for any minimal function $\pi$, it follows that $\pi(\tilde{p})>0$. Define the function $\pi^{\prime}$ by

$$
\pi^{\prime}(p):= \begin{cases}\frac{1}{1+\delta} \pi(\tilde{p}) & \text { if } p=\tilde{p} \\ \pi(p) & \text { if } p \neq \tilde{p}\end{cases}
$$

We show that $\pi^{\prime}$ is valid. Consider any $\bar{y} \in I_{S}(P)$ for some matrix $P$ containing column $\tilde{p}$. Note that

$$
\sum \pi^{\prime}(p) \bar{y}_{p}=\sum_{p \neq \tilde{p}} \pi(p) \bar{y}_{p}+\frac{1}{1+\delta} \pi(\tilde{p}) \bar{y}_{\tilde{p}} .
$$

If $\bar{y}_{\tilde{p}}=0$ then $\sum \pi^{\prime}(p) \bar{y}_{p}=\sum \pi(p) \bar{y}_{p} \geq 1$ because $\pi$ is valid. If $\bar{y}_{\tilde{p}} \geq(1+\delta) / \pi(\tilde{p})$ then $\sum \pi^{\prime}(p) \bar{y}_{p} \geq 1$. Thus we can assume that $1 \leq \bar{y}_{\tilde{p}}<(1+\delta) / \pi(\tilde{p})$.

Observe that $\sum_{p \neq \tilde{p}} \pi(p) \bar{y}_{p}+\pi(\tilde{p})\left(\bar{y}_{\tilde{p}}-1\right) \geq \sum_{p \neq \tilde{p}} \pi\left(p \bar{y}_{p}\right)+\pi\left(\tilde{p}\left(\bar{y}_{\tilde{p}}-1\right)\right) \geq \pi\left(\sum_{p \neq \tilde{p}} p \bar{y}_{p}+\right.$ $\left.\tilde{p}\left(\bar{y}_{\tilde{p}}-1\right)\right)=\pi(b-\tilde{p})$, where the inequalities follow by the subadditivity of $\pi$ and the equality follows as $\pi$ is periodic modulo $\mathbb{Z}^{n}$ and $\sum_{p \neq \tilde{p}} p \bar{y}_{p}+\tilde{p} \bar{y}_{\tilde{p}} \in S$. Therefore

$$
\begin{aligned}
\sum \pi^{\prime}(p) \bar{y}_{p} & =\sum_{p \neq \tilde{p}} \pi(p) \bar{y}_{p}+\pi(\tilde{p})\left(\bar{y}_{\tilde{p}}-1\right)+\pi(\tilde{p})-\frac{\delta}{1+\delta} \pi(\tilde{p}) \bar{y}_{\tilde{p}} \\
& \geq \pi(b-\tilde{p})+\pi(\tilde{p})-\delta \\
& =1+\delta-\delta=1 .
\end{aligned}
$$

This shows that $\pi^{\prime}$ is valid, contradicting the minimality of $\pi$.

We now prove the "if" part of the statement. Assume that $\pi(w)=0$ for all $w \in \mathbb{Z}^{n}, \pi$ is subadditive, and satisfies the symmetry condition. As noted earlier, the first two conditions imply that $\pi$ is periodic.

We first show that $\pi$ is valid. The symmetry condition implies $\pi(0)+\pi(b)=1$. Since $\pi(0)=0$, we have $\pi(b)=1$. Let $P$ and $\bar{y}$ be such that $P \bar{y}=b+w$ for some $w \in \mathbb{Z}^{n}$. We have that $\sum \pi(p) \bar{y}_{p} \geq \pi\left(\sum p \bar{y}_{p}\right)=\pi(b+w)=\pi(b)=1$, where the inequality comes from subadditivity and the second to last equality comes from periodicity. Thus $\pi$ is valid.

To show that $\pi$ is minimal, suppose by contradiction that there exists an integer valid function $\pi^{\prime} \leq \pi$ such that $\pi^{\prime}(\tilde{p})<\pi(\tilde{p})$ for some $\tilde{p} \in \mathbb{R}^{n}$. Then $\pi(\tilde{p})+\pi(b-\tilde{p})=1$ implies $\pi^{\prime}(\tilde{p})+\pi^{\prime}(b-\tilde{p})<1$, contradicting the validity of $\pi^{\prime}$.

The above proof follows the one given in [32].

\subsection{Extreme functions: Techniques for proving extremality}

When describing a full-dimensional polyhedron $K$, one is only interested in identifying the facet-defining inequalities of $K$, as all other valid inequalities can be expressed as convex combinations of facet-defining inequalities. In other words, the facet-defining inequalities form extreme rays of the cone of valid inequalities for $K$. In our context, the analogous notion is that of an extreme function. An integer valid function $\pi$ is extreme for $S$ if it cannot be written as a proper convex combination of two other integer valid functions for $S$, i.e., if $\pi=\frac{1}{2}\left(\pi^{1}+\pi^{2}\right)$ for integer valid functions $\pi^{1}, \pi^{2}$ implies $\pi=\pi^{1}=\pi^{2}$. Extreme functions are easily seen to be minimal. 
REMARK 5.2. For the continuous model, extreme functions are defined in the same way and were characterized by Cornuéjols and Margot [36] for the case $n=2$. See also [17, Theorem 1.5] for a result in general dimension $n$, which states that a valid function for the continuous model is extreme if and only if a certain restriction of this function gives a facet defining inequality for a well-defined polyhedron.

The following lemma will be useful in analyzing extreme functions.

Lemma 5.3. Let $S=b+\mathbb{Z}^{n}$ for some $b \notin \mathbb{Z}^{n}$. Let $\pi$ be a nonnegative minimal integer valid function for $S$ and suppose $\pi^{1}$ and $\pi^{2}$ are nonnegative integer valid functions such that $\pi=\frac{1}{2}\left(\pi^{1}+\pi^{2}\right)$. Then the following hold:

(i) $\pi^{1}, \pi^{2}$ are minimal [52].

(ii) Let the additivity domain of $\pi$ be:

$$
E(\pi):=\{(x, y): \pi(x)+\pi(y)=\pi(x+y)\} .
$$

Then $E(\pi) \subseteq E\left(\pi^{1}\right) \cap E\left(\pi^{2}\right)$ [52].

(iii) Suppose there exists a real number $M$ such that $\lim _{\sup _{h \rightarrow 0^{+}}} \frac{\pi(h r)}{h} \leq M$ for all $r \in \mathbb{R}^{n}$ such that $\|r\|=1$. Then $\pi$ is Lipschitz-continuous. Furthermore, this condition holds for $\pi^{1}$ and $\pi^{2}$, and $\pi^{1}, \pi^{2}$ are Lipschitz-continuous [23, Theorem 2.9].

To prove that a function $\pi$ is extreme, the main idea is to establish that $E(\pi) \subseteq E\left(\pi^{\prime}\right)$ implies $\pi=\pi^{\prime}$ for every minimal integer valid function $\pi^{\prime}$. Then, starting from the assumption $\pi=\frac{1}{2}\left(\pi^{1}+\pi^{2}\right)$, by Lemma 5.3 (ii) $E(\pi) \subseteq E\left(\pi^{1}\right)$ and $E(\pi) \subseteq E\left(\pi^{2}\right)$, and therefore $\pi=\pi^{1}=$ $\pi^{2}$.

The main tools for establishing that $E(\pi) \subseteq E\left(\pi^{\prime}\right)$ implies $\pi=\pi^{\prime}$ for every minimal integer valid function $\pi^{\prime}$ are results that are collectively called interval lemmas (after the one-dimensional interval lemma of Gomory and Johnson [54]), and are presented in the next subsection.

We illustrate this framework for proving extremality by outlining a proof of the $(n+1)$ slope theorem of Basu, Hildebrand, Köppe and Molinaro [24], which is one of the most general sufficient conditions for extremality of minimal integer valid functions.

\subsubsection{Regular solutions to Cauchy's functional equation}

As mentioned above, the key to establishing extremality of a function $\pi$ is to prove that $E(\pi) \subseteq E\left(\pi^{\prime}\right)$ implies $\pi=\pi^{\prime}$ for every minimal integer valid function $\pi^{\prime}$. The first step is to show that $E(\pi) \subseteq E\left(\pi^{\prime}\right)$ implies that $\pi^{\prime}$ has an affine linear structure whenever $\pi$ has such structure. For this purpose, we consider full-dimensional convex subsets $F \subseteq E\left(\pi^{\prime}\right) \subseteq$ $\mathbb{R}^{n} \times \mathbb{R}^{n}$; therefore, $\pi(u)+\pi(v)=\pi(u+v)$ for all $(u, v) \in F$. This leads to the study of functions $\theta: \mathbb{R}^{n} \rightarrow \mathbb{R}$ satisfying:

$$
\theta(u)+\theta(v)=\theta(u+v), \quad(u, v) \in F
$$

for a given subset $F \subseteq \mathbb{R}^{n} \times \mathbb{R}^{n}$. This equation is known as the (additive) Cauchy functional equation. 
The Cauchy functional equation is classically studied for functions $\theta: \mathbb{R} \rightarrow \mathbb{R}$, where the additivity domain $F$ is the entire space $\mathbb{R} \times \mathbb{R}$ (see, e.g., [1]). In addition to the regular solutions, which are the (homogeneous) linear functions $\theta(x)=c x$ for any $c \in \mathbb{R}$, there exist certain pathological solutions, which are highly discontinuous [1, Chapter 2, Lemma 3]. In order to rule out these solutions, one imposes a regularity hypothesis. Various such regularity hypotheses have been proposed in the literature; for example, it is sufficient to assume that the function $\theta$ is bounded on every bounded interval [1, Chapter 2, Theorem 8].

We now return to functions $\theta: \mathbb{R}^{n} \rightarrow \mathbb{R}$ and recall the notion of affine functions over a domain.

Definition 5.4. Let $U \subseteq \mathbb{R}^{n}$. We say that $\theta: U \rightarrow \mathbb{R}$ is affine over $U$ (with gradient $c$ ) if there exists $c \in \mathbb{R}^{n}$ such that for any $u_{1}, u_{2} \in U$ we have

$$
\theta\left(u_{2}\right)-\theta\left(u_{1}\right)=c\left(u_{2}-u_{1}\right) .
$$

Equivalently, there exists $b \in \mathbb{R}$ such that $\theta(u)=c u+b$ for every $u \in U$.

We define three projection operators on $\mathbb{R}^{n} \times \mathbb{R}^{n}$. For any subset $F \subseteq \mathbb{R}^{n} \times \mathbb{R}^{n}$, define $p_{1}(F)=\left\{u \in \mathbb{R}^{n}:(u, v) \in F\right\}, p_{2}(F)=\left\{v \in \mathbb{R}^{n}:(u, v) \in F\right\}$, and $p_{3}(F)=\{u+v:(u, v) \in$ $F\}$.

Lemma 5.5 (Convex additivity domain lemma). [21, Theorem 2.11] Let $\theta: \mathbb{R}^{n} \rightarrow \mathbb{R}$ be a bounded function. Let $F \subseteq \mathbb{R}^{n} \times \mathbb{R}^{n}$ be a full-dimensional convex set such that $\theta(u)+\theta(v)=$ $\theta(u+v)$ for all $(u, v) \in F$. Then there exists a vector $c \in \mathbb{R}^{n}$ such that $\theta$ is affine with the same gradient c over $\operatorname{int}\left(p_{1}(F)\right)$, $\operatorname{int}\left(p_{2}(F)\right)$ and $\operatorname{int}\left(p_{3}(F)\right)$, respectively.

The special case of the above result in which $F=U \times V$ is the cartesian product of two closed proper intervals $U, V \subseteq \mathbb{R}$ is the so-called interval lemma stated in [54] (see also [34, Lemma 6.26]). Early extensions to this original result were made in Dey and Richard's work [41, Proposition 23] and Dey et al's work [42, Proposition 3, Corollary 1].

\subsection{Sufficient conditions for extremality: the $(n+1)$-slope theorem}

One of the most celebrated results in the study of extreme functions is the so-called GomoryJohnson 2-slope theorem [52] (see also [34, Theorem 6.27]), which states that for $n=1$, if a continuous piecewise linear minimal valid integer function has only 2 values for the derivative wherever it exists ( 2 slopes), then the function is extreme. This was generalized to $n=2$ by Cornuéjols and Molinaro [37, and to general $n$ by Basu, Hildebrand, Köppe and Molinaro [24]. We present the general $(n+1)$-slope theorem here, along with the main ingredients of its proof.

We introduce the definition of polyhedral complex, a classical notion from polyhedral geometry [72, Chapter 5], to formalize the notion of piecewise linear functions over $\mathbb{R}^{n}$ for $n \geq 2$.

Definition 5.6. A polyhedral complex in $\mathbb{R}^{n}$ is a collection $\mathcal{P}$ of polyhedra in $\mathbb{R}^{n}$ such that:

(i) if $I \in \mathcal{P}$, then all faces of $I$ are in $\mathcal{P}$,

(ii) the intersection $I \cap J$ of two polyhedra $I, J \in \mathcal{P}$ is a face of both $I$ and $J$, 
(iii) any compact subset of $\mathbb{R}^{n}$ intersects only finitely many polyhedra in $\mathcal{P}$.

A polyhedron $I$ from $\mathcal{P}$ is called a face of the complex. We call the maximal faces of $\mathcal{P}$ the cells of $\mathcal{P}$. A function $\pi: \mathbb{R}^{n} \rightarrow \mathbb{R}$ is continuous piecewise linear if there exists a polyhedral complex $\mathcal{P}$ such that $\cup_{I \in \mathcal{P}} I=\mathbb{R}^{n}$ and $\pi$ is an affine function over each of the cells of $\mathcal{P}$ (thus automatically imposing continuity for the function).

We next define genuinely $n$-dimensional functions on $\mathbb{R}^{n}$ and then indicate that for the analysis of minimal and extreme functions, it suffices to study genuinely $n$-dimensional functions. This notion was first introduced in [24].

Definition 5.7. A function $\theta: \mathbb{R}^{n} \rightarrow \mathbb{R}$ is genuinely $n$-dimensional if there does not exist a function $\varphi: \mathbb{R}^{n-1} \rightarrow \mathbb{R}$ and a linear map $f: \mathbb{R}^{n} \rightarrow \mathbb{R}^{n-1}$ such that $\theta=\varphi \circ f$.

Remark 5.8 (Dimension reduction; [21, Proposition B.9, Remark B.10]). The extremality/minimality question for $\pi$ that is not genuinely $n$-dimensional can be reduced to the same question for a lower-dimensional genuinely $\ell$-dimensional function $($ so $\ell<n$.) When $\mathcal{P}$ is a rational polyhedral complex, this reduction can be done algorithmically. Thus, we can assume without loss of generality that a function is genuinely $n$-dimensional.

We can now state the $(n+1)$-slope theorem.

Theorem 5.9 ([24, Theorem 1.7]). Let $\pi: \mathbb{R}^{n} \rightarrow \mathbb{R}$ be a nonnegative minimal integer valid function that is continuous piecewise linear and genuinely $n$-dimensional with at most $n+1$ slopes, i.e., at most $n+1$ different values for the gradient of $\pi$ where it exists. Then $\pi$ is extreme and has exactly $n+1$ slopes.

We outline the proof of Theorem [5.9, Let $\pi$ be a continuous piecewise linear minimal integer valid function that is genuinely $n$-dimensional with at most $n+1$ slopes. Let $\mathcal{P}$ be the polyhedral complex associated with $\pi$.

1. Subadditivity and the genuine $n$-dimensionality of $\pi$ imply that $\pi$ has exactly $n+1$ gradient values $\bar{g}^{1}, \ldots, \bar{g}^{n+1} \in \mathbb{R}^{n}$. This is a relatively easy step, see Lemma 2.11 in [24].

2. (Compatibility step) Let $\pi^{1}, \pi^{2}$ be valid functions such that $\pi=\frac{1}{2}\left(\pi^{1}+\pi^{2}\right)$. For each $i=1, \ldots, n+1$, define $\mathcal{P}_{i} \subseteq \mathcal{P}$ to be the polyhedral complex formed by all the cells (and their faces) of $\mathcal{P}$ where the gradient of $\pi$ is $\bar{g}^{i}$. Show that there exist $\tilde{g}^{1}, \ldots, \tilde{g}^{n+1}$ such that $\pi^{1}$ is affine over every cell in $\mathcal{P}_{i}$ with gradient $\tilde{g}^{i}$.

3. (Gradient matching step) We then prove properties of genuinely $n$-dimensional functions with $n+1$ slopes that lead to a system of equations that are satisfied by the coefficients of $\bar{g}^{1}, \ldots, \bar{g}^{n+1}$ and $\tilde{g}^{1}, \ldots, \tilde{g}^{n+1}$. Then, it is established that this system of equations has a unique solution, and thus, $\bar{g}^{i}=\tilde{g}^{i}$ for every $i=1, \ldots, n+1$.

4. For every $r \in \mathbb{R}^{n}$ there exist $\mu_{1}, \mu_{2}, \ldots, \mu_{n+1}$ such that $\mu_{i}$ is the fraction of the segment $[0, r]$ that lies in $\mathcal{P}_{i}$. Thus,

$$
\pi(r)=\pi(0)+\sum_{i=1}^{n+1} \mu_{i}\left(\bar{g}^{i} r\right)=\pi^{1}(0)+\sum_{i=1}^{n+1} \mu_{i}\left(\tilde{g}^{i} r\right)=\pi^{1}(r) .
$$

This proves that $\pi=\pi^{1}$ and thus, $\pi=\pi^{1}=\pi^{2}$, concluding the proof of Theorem 5.9,

We now elaborate on Steps 2. and 3. 
Compatibility step. The analysis of step 1 also shows that for every $i=1, \ldots, n+1$, there exist $C_{i} \in \mathcal{P}_{i}$ such that $0 \in C_{i}$. This means that for every gradient value, there is a cell containing the origin with that gradient. Fix an arbitrary $i \in\{1, \ldots, n+1\}$ and consider any cell $I \in \mathcal{P}_{i}$. Let $F=\left\{(u, v) \in \mathbb{R}^{n} \times \mathbb{R}^{n}: u \in C_{i}, v \in I, u+v \in I\right\}$. Then $F \subseteq E(\pi)$ since for $(u, v)$ such that $u \in C_{i}, v \in I, u+v \in I, \pi(u)+\pi(v)-\pi(u+v)=$ $\left(\bar{g}^{i} u\right)+\left(\bar{g}^{i} v+\delta\right)-\left(\bar{g}^{i}(u+v)+\delta\right)=0$ for some $\delta \in \mathbb{R}$; here, we use the fact that $\pi$ is affine over $C_{i}$ and $I$ with gradient $g^{i}$, and the facts that $0 \in C_{i}$ and $\pi(0)=0$. By Lemma 5.3 (ii), $F \subseteq E\left(\pi^{1}\right)$; by Lemma 5.3 (iii), $\pi^{1}$ is continuous (because $\pi$ satisfies the hypothesis of Lemma 5.3 (iii) as $\pi$ is continuous piecewise linear). By Lemma 5.5, applied to $F$ and $\theta=\pi^{1}$, and continuity of $\pi^{1}$, we obtain that $\pi^{1}$ is affine on $C_{i}=p_{1}(F)$ and $I=p_{2}(F)=p_{3}(F)$ with the same gradient. Since the choice of $I$ was arbitrary, this establishes that for every cell $I \in \mathcal{P}_{i}, \pi^{1}$ is affine with the same gradient; this is precisely the desired $\tilde{g}^{i}$.

Gradient matching step. The system of equations of step 3 has two sets of constraints, the first of which follows from the condition that $\pi(b+w)=1$ for every $w \in \mathbb{Z}^{n}$ (see Theorem 5.1). The second set of constraints is more involved. Consider two adjacent cells $I, I^{\prime} \in \mathcal{P}$ that contain a segment $[x, y] \subseteq \mathbb{R}^{n}$ in their intersection. Along the line segment $[x, y]$, the gradients of $I$ and $I^{\prime}$ projected onto the line spanned by the vector $y-x$ must agree; the second set of constraints captures this observation. We will identify a set of vectors $r^{1}, \ldots, r^{n+1}$ such that every subset of $n$ vectors is linearly independent and such that each vector $r^{i}$ is contained in $n$ cells of $\mathcal{P}$ with different gradients. We then use the segment $\left[0, r^{i}\right]$ to obtain linear equations involving the gradients of $\pi$ and $\pi^{\prime}$. The fact that every subset of $n$ vectors is linearly independent will be crucial in ensuring the uniqueness of the solution to the system of equations.

Lemma 5.10 ([24, Lemma 3.10]). There exist vectors $r^{1}, r^{2}, \ldots, r^{n+1} \in \mathbb{R}^{n}$ with the following properties:

(i) For every $i, j, \ell \in\{1, \ldots, n+1\}$ with $j, \ell$ different from $i$, the equations $r^{i} \bar{g}^{j}=r^{i} \bar{g}^{\ell}$ and $r^{i} \tilde{g}^{j}=r^{i} \tilde{g}^{\ell}$ hold.

(ii) Every $n$-subset of $\left\{r^{1}, \ldots, r^{n+1}\right\}$ is linearly independent.

The proof of Lemma 5.10 uses a result known as the Knaster-Kuratowski-Mazurkiewicz Lemma (KKM Lemma) from fixed point theory, which exposes a nice structure in the gradient pattern of $\pi$.

Lemma 5.11 (KKM [60, 2]). Consider a simplex $\operatorname{conv}\left(u^{j}\right)_{j=1}^{d}$. Let $F_{1}, F_{2}, \ldots, F_{d}$ be closed sets such that for all $J \subseteq\{1, \ldots, d\}$, the face $\operatorname{conv}\left(u^{j}\right)_{j \in J}$ is contained in $\bigcup_{j \in J} F_{j}$. Then the intersection $\bigcap_{j=1}^{d} F_{j}$ is non-empty.

This lemma is applied to the facets of a certain simplex $\Delta$ containing the origin, and the closed sets $F_{i}=\bigcup_{I \in \mathcal{P}_{i}}(\Delta \cap I)$, for $i=1, \ldots, n+1$. For each facet of $\Delta$ indexed by $i=1, \ldots, n+1$, the KKM lemma (with $d=n$ ) ensures the existence of a point $r^{i} \in \bigcap_{j \neq i} F_{j}$ on the facet indexed by $i$. These points give the vectors $r^{1}, \ldots, r^{n+1}$ from Lemma 5.10. The bulk of the technicality lies in proving that the chosen simplex and the sets $F_{i}$ satisfy the hypothesis of the KKM lemma.

We finally present the system of linear equations that we consider. 
Corollary 5.12 ([24, Corollary 3.13]). Consider any $n+1$ affinely independent vectors $a^{1}, a^{2}, \ldots, a^{n+1} \in b+\mathbb{Z}^{n}$. Also, let $r^{1}, r^{2}, \ldots, r^{n+1}$ be the vectors given by Lemma 5.10. Then there exist $\mu_{i j} \in \mathbb{R}_{+}$for $i, j \in\{1, \ldots, n+1\}$, with $\sum_{j=1}^{n+1} \mu_{i j}=1$ for all $i \in\{1, \ldots, n+1\}$, such that both $\tilde{g}^{1}, \ldots, \tilde{g}^{n+1}$ and $\bar{g}^{1}, \ldots, \bar{g}^{n+1}$ are solutions to the linear system

$$
\begin{aligned}
\sum_{j=1}^{n+1}\left(\mu_{i j} a^{i}\right) g^{j}=1 & \text { for all } i \in\{1, \ldots, n+1\}, \\
r^{i} g^{j}-r^{i} g^{\ell}=0 & \text { for all } i, j, \ell \in\{1, \ldots, n+1\} \text { such that } i \neq j, \ell,
\end{aligned}
$$

with variables $g^{1}, \ldots, g^{n+1} \in \mathbb{R}^{n}$.

We remark that we can always find vectors $a^{1}, a^{2}, \ldots, a^{n+1} \in b+\mathbb{Z}^{n}$ such that the set $a^{1}, \ldots, a^{n+1}$ is affinely independent, so the system above indeed exists. Property (ii) in Lemma 5.10 and the fact that $a^{1}, \ldots, a^{n+1}$ are affinely independent can be leveraged to show that (5.4) has either no solutions or a unique solution. However, the linear algebra is involved and we refer the reader to [24, Section 3.3]. Since $\bar{g}^{1}, \ldots, \bar{g}^{n+1}$ is a solution, the conclusion is that the system has a unique solution.

\section{The mixed integer model}

We consider here the mixed integer model (2.1):

$$
X_{S}(R, P):=\left\{(s, y) \in \mathbb{R}_{+}^{k} \times \mathbb{Z}_{+}^{\ell}: R s+P y \in S\right\}
$$

where $S \subseteq \mathbb{R}^{n} \backslash\{0\}$ is a closed set, and $k$ and $\ell$ are both positive. Recall that a pair of functions $(\psi, \pi)$ is a valid pair if and only if $\sum \psi(r) s_{r}+\sum \pi(p) y_{p} \geq 1$ is a valid inequality for $X_{S}(R, P)$, for every $k, \ell, R$ and $P$. We do not make any nonnegativity assumptions on the functions $\psi, \pi$ in this section, as was done in Section 5. Note that if $(\psi, \pi)$ is a valid pair, then $\psi$ is a valid function and $\pi$ is an integer valid function. However, the converse does not hold.

When $S=b+\mathbb{Z}^{n}$, Theorem 4.4 characterizes minimal valid functions $\psi$ for the continuous model, and Theorem 5.1 characterizes nonnegative minimal integer valid functions $\pi$ for the pure integer model. For the mixed integer model (2.1), Johnson [58] gives such a characterization for nonnegative minimal valid pairs.

TheOrem 6.1. Let $S=b+\mathbb{Z}^{n}$ for some $b \notin \mathbb{Z}^{n}$, and let $(\psi, \pi)$ be a valid pair, with $\pi \geq 0$. Then $(\psi, \pi)$ is a minimal valid pair if and only if $\pi$ is a minimal integer valid function and $\psi$ satisfies

$$
\psi(r)=\limsup _{h \rightarrow 0^{+}} \frac{\pi(h r)}{h} \quad \text { for every } r \in \mathbb{R}^{n} .
$$

When $S=b+\mathbb{Z}^{n}$, for the continuous model, Theorem 4.4 shows that minimal functions $\psi$ are gauge functions of $K$, where polyhedron $K$ is a maximal $S$-free convex set, and Theorem 4.5 gives a formula for the computation of $\psi$. One of the most studied procedures to compute a minimal pair $(\psi, \pi)$ for the mixed integer model is to start from a minimal function $\psi$ for the continuous model, and then compute $\pi$ such that $(\psi, \pi)$ is a minimal pair. Such a 
procedure goes under that name of lifting. A lifting of a valid function $\psi$ is a function $\pi$ such that $(\psi, \pi)$ is a valid pair. A lifting $\pi$ of $\psi$ is minimal if $\pi=\pi^{\prime}$ for every lifting $\pi^{\prime}$ of $\psi$ such that $\pi^{\prime} \leq \pi$.

Theorem 6.1 shows that if $(\psi, \pi)$ is a minimal valid pair and $\pi \geq 0$, then $\pi$ is a minimal integer valid function. However, $\psi$ is not guaranteed to be a minimal valid function. Therefore the lifting procedure outlined above, applied to a minimal valid function, only produces a subset of minimal valid pairs, which however includes the most well-known and computationally effective pairs.

The idea of using lifting in this context was proposed by Dey and Wolsey [44, who imported the concept of monoidal strengthening. Monoidal strengthening was introduced by Balas and Jeroslow [13] to strengthen cutting planes by using integrality information. Searching for minimal liftings $\pi$ is analogous to the idea of strengthening the "trivial" valid pair $(\psi, \psi)$ by using the integrality information on the $y$ variables.

\subsection{A geometric view of lifting}

The following proposition imposes regularity on the structure of minimal liftings.

Proposition 6.2 ([25, Proposition A.3]). Given a closed set $S \subseteq \mathbb{R}^{n} \backslash\{0\}$, let $\psi$ be a valid function and $\pi$ a minimal lifting of $\psi$. Define

$$
W_{S}:=\left\{w \in \mathbb{R}^{n}: s+\lambda w \in S, \forall s \in S, \forall \lambda \in \mathbb{Z}\right\} .
$$

Then $\pi(p+w)=\pi(p)$ for all $p \in \mathbb{R}^{n}$ and $w \in W_{S}$ (i.e., $\pi$ is periodic modulo $W_{S}$ ).

Proof. Let $\pi$ be a minimal lifting of $\psi$. Assume to the contrary that there exists some $\hat{p} \in \mathbb{R}^{n}$ and $w \in W_{S}$ such that $\pi(\hat{p}) \neq \pi(\hat{p}+w)$. Since $-w \in W_{S}$, we may assume $\pi(\hat{p})>\pi(\hat{p}+w)$. Define a function $\tilde{\pi}$ as $\tilde{\pi}(\hat{p})=\pi(\hat{p}+w)$ and $\tilde{\pi}(p)=\pi(p)$ for $p \neq \hat{p}$. We show that $\tilde{\pi}$ is a lifting of $\psi$, a contradiction to the assumption that $\pi$ is a minimal lifting.

Let $R \in \mathbb{R}^{n \times k}, P \in \mathbb{R}^{n \times \ell}$, and $(s, y) \in X_{S}(R, P)$. We assume, without loss of generality, that $\hat{p}$ and $\hat{p}+w$ are columns of $P$ and we show that $\sum \psi(r) s_{r}+\sum \tilde{\pi}(p) y_{p} \geq 1$. Let $\bar{y}$ be such that $\bar{y}_{\hat{p}}=0$ and $\bar{y}_{p}=y_{p}$ for $p \neq \hat{p}$. Since $w \in W_{S}$ and $y_{\hat{p}} \in \mathbb{Z}$, it follows that

$$
R s+P y \in S \Longleftrightarrow R s+P y+w y_{\hat{p}}=R s+P \bar{y}+(\hat{p}+w) y_{\hat{p}} \in S .
$$

Let $y^{\prime}$ be defined as $y_{\hat{p}+w}^{\prime}=y_{\hat{p}}+y_{\hat{p}+w}, y_{\hat{p}}^{\prime}=0$ and $y_{p}^{\prime}=y_{p}$ for $p \neq \hat{p}+w, \hat{p}$. Since $\pi$ is a lifting of $\psi$ and $R s+P y^{\prime}=R s+P \bar{y}+(\hat{p}+w) y_{\hat{p}} \in S$, we have

$$
\sum \psi(r) s_{r}+\sum \tilde{\pi}(p) y_{p}=\sum \psi(r) s_{r}+\sum \pi(p) y_{p}^{\prime} \geq 1 .
$$

The set $W_{S}$ for arbitrary $S$ was defined first in [25], where the above proposition is also proved.

Given a valid function $\psi$, the lifting region $T_{\psi}$ (first introduced in [44]) is:

$$
T_{\psi}:=\left\{r \in \mathbb{R}^{n}: \pi(r)=\psi(r) \text { for every minimal lifting } \pi \text { of } \psi\right\} .
$$

Conforti et al. 33] prove that: 
TheOREM 6.3. If $S=\left(b+\mathbb{Z}^{n}\right) \cap Q$, where $b \notin \mathbb{Z}^{n}$ and $Q$ is a rational polyhedron, and $\psi$ is a minimal valid function, then there exists a full-dimensional ball $B(0, \varepsilon) \subseteq T_{\psi}$ for some $\varepsilon>0$.

The next theorem provides a lifting for any valid function $\psi$ and characterizes a condition on $T_{\psi}$ for this lifting to be minimal.

Theorem 6.4 ([25, Proposition A.3]). Given a closed set $S \subseteq \mathbb{R}^{n} \backslash\{0\}$, let $\psi$ be a valid function and define

$$
\pi^{*}(p)=\inf _{w \in W_{S}} \psi(p+w), \text { for all } p \in \mathbb{R}^{n} .
$$

Then $\pi^{*}$ is a lifting of $\psi$. Moreover, if $T_{\psi}+W_{S}=\mathbb{R}^{n}$, then $\pi^{*}$ is a minimal lifting of $\psi$ and $\pi^{*}(p)=\psi(p+w)$ for any $w \in W_{S}$ such that $p+w \in T_{\psi}$.

Proof. We first show that $\left(\psi, \pi^{*}\right)$ is a valid pair. Suppose to the contrary that there exist matrices $R, P$ and $(\bar{s}, \bar{y}) \in X_{S}(R, P)$ such that $\sum \psi(r) \bar{s}_{r}+\sum \pi^{*}(p) \bar{y}_{p}<1$. Let $\varepsilon=1-$ $\sum \psi(r) \bar{s}_{r}-\sum \pi^{*}(p) \bar{y}_{p}$. For each column $p$ of the matrix $P$, by definition of $\pi^{*}$, there exists $w_{p} \in W_{S}$ such that $\psi\left(p+w_{p}\right) \leq \pi^{*}(p)+\frac{\varepsilon}{2 \ell\left(\bar{y}_{p}+1\right)}$ (recall that $\ell$ is the number of columns of $P)$. Let $P^{\prime}$ be the matrix with columns $p+w_{p}$. Then $R \bar{s}+P^{\prime} \bar{y}=R \bar{s}+P \bar{y}+W \bar{y}$, where $W$ is the matrix with columns $w_{p}$. Since $W \bar{y} \in W_{S},(\bar{s}, \bar{y}) \in X_{S}\left(R, P^{\prime}\right)$. However,

$$
\begin{aligned}
1 \leq \sum \psi(r) \bar{s}_{r}+\sum \psi\left(p+w_{p}\right) \bar{y}_{p} & \leq \sum \psi(r) \bar{s}_{r}+\sum\left(\pi^{*}(p)+\frac{\varepsilon}{2 \ell\left(\bar{y}_{p}+1\right)}\right) \bar{y}_{p} \\
& \leq \sum \psi(r) \bar{s}_{r}+\sum \pi^{*}(p) \bar{y}_{p}+\frac{\varepsilon}{2} \\
& =1-\varepsilon+\frac{\varepsilon}{2}<1
\end{aligned}
$$

where we used the fact that $(\psi, \psi)$ is a valid pair. Thus we have a contradiction, and therefore $\pi^{*}$ is a lifting of $\psi$.

Let $\pi$ be any minimal lifting. Consider any $p \in \mathbb{R}^{n}$ and let $w \in W_{S}$ be such that $p+w \in T_{\psi}$. By Proposition 6.2, $\pi(p)=\pi(p+w)=\psi(p+w) \geq \pi^{*}(p)$. This implies that $\pi(p)=\pi^{*}(p)=\psi(p+w)$ since $\pi$ is a minimal lifting.

Importance of the lifting region. In light of the above results, if we start with a valid function $\psi$ whose values we can compute, and an explicit description for $T_{\psi}$ can be obtained, then the coefficients $\pi^{*}(p)$ can be computed by finding a $w \in W_{S}$ such that $p+w \in T_{\psi}$, and then using the formula for $\psi(p+w)$. Moreover, if $\psi$ is a minimal valid function, then $(\psi, \pi)$ is a minimal valid pair. Basu et al. [16] show that when $S=\left(b+\mathbb{Z}^{n}\right) \cap Q$ where $Q$ is a rational polyhedron, $T_{\psi}$ can be described as the finite union of full dimensional polyhedra, each of which has an explicit inequality description. This is discussed below.

\subsubsection{A description of the lifting region}

Throughout this section we assume $S=\left(b+\mathbb{Z}^{n}\right) \cap Q$ where $Q$ is a rational polyhedron, and we discuss the lifting region $T_{\psi}$ of a minimal valid function $\psi$. Recall from Theorem 4.22 that in this case maximal $S$-free convex sets are polyhedra. Let $a_{i} r \leq 1, i \in I$ be an irredundant description of a maximal $S$-free polyhedron $K$ with $0 \in \operatorname{int}(K)$. Recall that the minimal valid function $\psi$ associated with $K$ is $\psi(r)=\max _{i \in I} a_{i} r$. 
For each $s \in K \cap S$, let $k(s) \in I$ be an index such that $a_{k(s)} s=1$. By Theorem 4.22 such an index exists since $K$ is a maximal $S$-free convex set, and $s$ is on the boundary of $K$. Define the spindle $T(s)$ as follows:

$$
T(s):=\left\{r \in \mathbb{R}^{n}:\left(a_{i}-a_{k(s)}\right) r \leq 0,\left(a_{i}-a_{k(s)}\right)(s-r) \leq 0 \text { for all } i \in I\right\} .
$$

Basu et al. [16] prove the following:

Theorem 6.5. Let $S=\left(b+\mathbb{Z}^{n}\right) \cap Q$, where $b \notin \mathbb{Z}^{n}$ and $Q$ is a rational polyhedron, and let $K$ and $\psi$ be as above. Then the lifting region is:

$$
T_{\psi}=T(S, K):=\bigcup_{s \in K \cap S} T(s)
$$

Figure 2 illustrates the region $T_{\psi}$ for several examples. We collect some basic properties of the lifting region that were presented in [16]. Define $L_{K}=\left\{r \in \mathbb{R}^{n}: a_{i} r=a_{j} r\right.$ for all $\left.i, j \in I\right\}$.

Proposition 6.6. Assume $S=\left(b+\mathbb{Z}^{n}\right) \cap Q$, where $b \notin \mathbb{Z}^{n}$ and $Q$ is a rational polyhedron, and let $K$ be a maximal $S$-free polyhedron with $0 \in \operatorname{int}(K)$. The following hold:

(i) $\operatorname{lin}(T(s))=\operatorname{rec}(T(s))=L_{K}$ for every $s \in K \cap S$.

(ii) $T(s)=T\left(s^{\prime}\right)$ for every $s, s^{\prime} \in \mathbb{R}^{n}$ such that $s-s^{\prime} \in L_{K}$.

(iii) $T(S, K)$ is a union of finitely many polyhedra.

Proof. (i) From the description of $T(s)$,

$$
\operatorname{rec}(T(s))=\left\{r \in \mathbb{R}^{n}:\left(a_{i}-a_{k(s)}\right) r \leq 0, \quad\left(a_{k(s)}-a_{i}\right) r \leq 0 \text { for all } i \in I\right\}
$$

where $k(s)$ is the index of the facet of $K$ containing $s$. Hence, for every $r \in \operatorname{rec}(T(s))$, we obtain $a_{i} r=a_{k(s)} r$ for all $i \in I$. This shows that $\operatorname{rec}(T(s))=\operatorname{lin}(T(s))=L_{K}$.

(ii) Observe that

$$
\begin{aligned}
r \in T\left(s^{\prime}\right) & \Leftrightarrow\left(a_{i}-a_{k\left(s^{\prime}\right)}\right) r \leq 0, \quad\left(a_{i}-a_{k\left(s^{\prime}\right)}\right)\left(s^{\prime}-r\right) \leq 0 \quad \forall i \in I \\
& \Leftrightarrow\left(a_{i}-a_{k\left(s^{\prime}\right)}\right) r \leq 0, \quad\left(a_{i}-a_{k\left(s^{\prime}\right)}\right)\left(s^{\prime}+\left(s-s^{\prime}\right)-r\right) \leq 0 \quad \forall i \in I \\
& \Leftrightarrow\left(a_{i}-a_{k(s)}\right) r \leq 0, \quad\left(a_{i}-a_{k(s)}\right)(s-r) \leq 0 \quad \forall i \in I
\end{aligned}
$$

where the second equivalence follows from the fact that $s-s^{\prime} \in L_{K}$ and so $a_{i}\left(s-s^{\prime}\right)=$ $a_{k}\left(s-s^{\prime}\right)$, and in the third equivalence we use the fact that $k(s)$ and $k\left(s^{\prime}\right)$ can be chosen to be the same as $s-s^{\prime} \in L_{K}$.

(iii) Since $K$ is full-dimensional, $K$ satisfies either condition (i) or condition (ii) of Theorem 4.22. In both cases, if we define $L:=\langle\operatorname{rec}(K \cap \operatorname{conv}(S))\rangle$, then $L$ is a lattice subspace contained in $\operatorname{lin}(K)$. Since $\operatorname{lin}(K)=\left\{r \in \mathbb{R}^{n}: a_{i} r=0\right.$ for all $\left.i \in I\right\}$, we have that $L \subseteq \operatorname{lin}(K) \subseteq L_{K}$

Since $L=\langle\operatorname{rec}(K \cap \operatorname{conv}(S))\rangle$, it follows that $\operatorname{proj}_{L^{\perp}}(K) \cap \operatorname{proj}_{L^{\perp}}(\operatorname{conv}(S))$ is a polytope. 


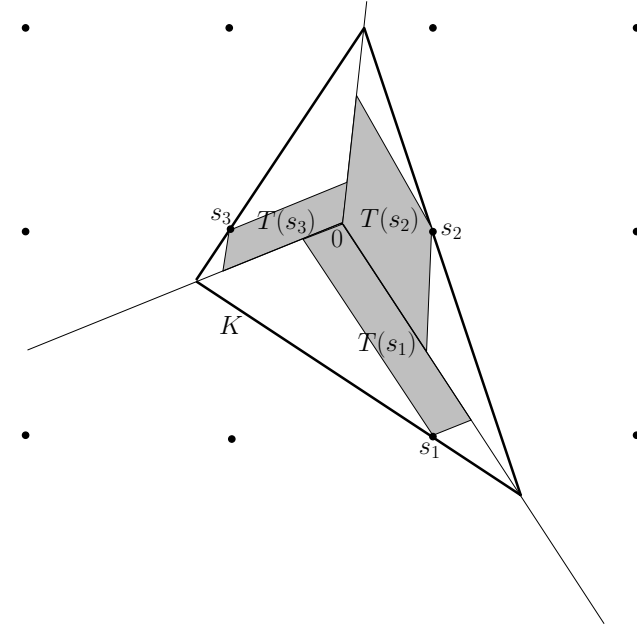

(a) A maximal $\left(b+\mathbb{Z}^{2}\right)$-free triangle with three integer points

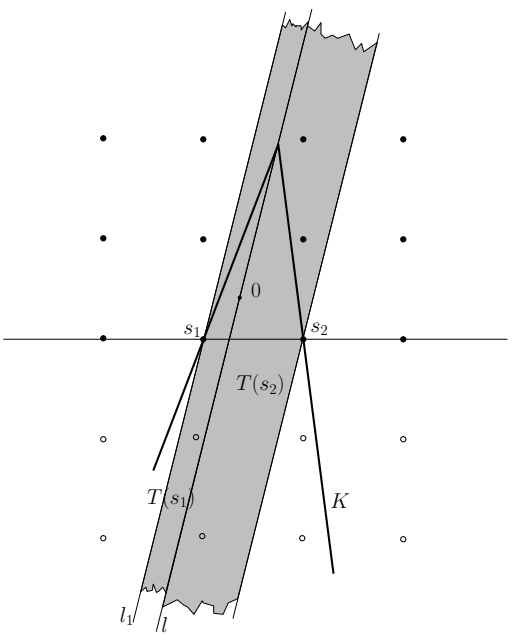

(c) A wedge

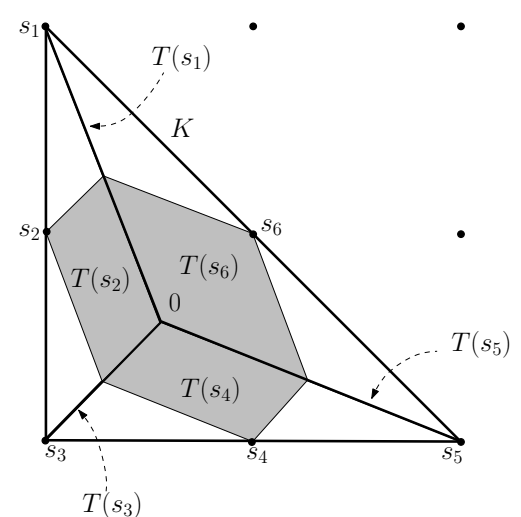

(b) A maximal $\left(b+\mathbb{Z}^{2}\right)$-free triangle with integer vertices

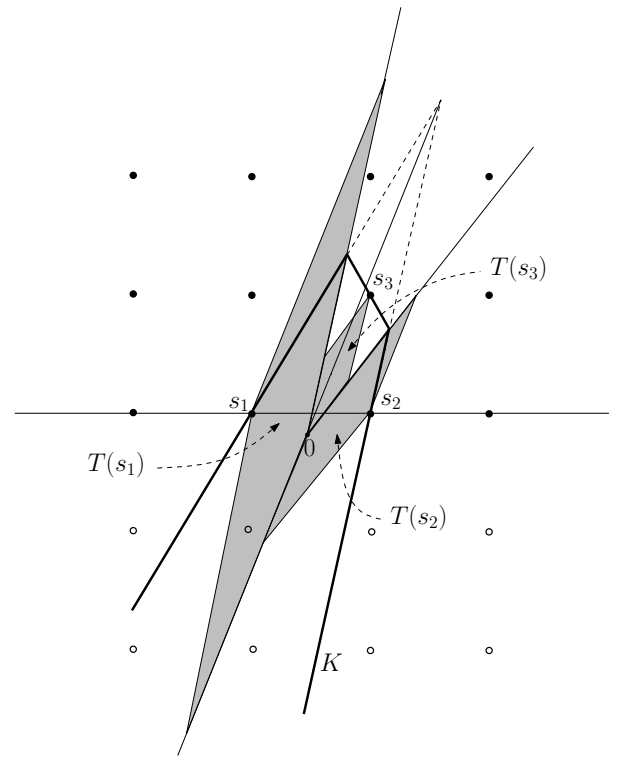

(d) A truncated wedge

Figure 2: Regions $T(s)$ for some maximal $S$-free polyhedra $K$ in the plane and $s \in S \cap K$. In Figures $2(\mathrm{a})$ and $2(\mathrm{~b}), S=b+\mathbb{Z}^{2}$ for some $b \notin \mathbb{Z}^{2}$ and in Figures $2(\mathrm{c})$ and $2(\mathrm{~d}) S=\left(b+\mathbb{Z}^{2}\right) \cap H$ where $H$ is a half-space shown in the figures (dark circles show points from $S$ and hollow circles show points in $b+\mathbb{Z}^{2}$ that are not in $S$ ). The thick dark line indicates the boundary of $K$. For a particular $s$, the dark gray regions denote $T(s)$. The jagged lines in a region indicate that it extends to infinity. For example, in Figure 2(c), $T\left(s_{1}\right)$ is the strip between lines $l_{1}$ and $l$. Figure 2(b) shows an example where $T(s)$ is full-dimensional for $s_{2}, s_{4}, s_{6}$, but is not full-dimensional for $s_{1}, s_{3}, s_{5}$. 
Given two elements $s, s^{\prime} \in S$ whose orthogonal projections onto $L^{\perp}$ coincide, it follows that $s-s^{\prime} \in L \subseteq L_{K}$, and therefore by (ii) $T(s)=T\left(s^{\prime}\right)$. Then the number of sets $T(s)$, $s \in S \cap K$, is at most the cardinality of $\operatorname{proj}_{L^{\perp}}(S \cap K)$.

Let $S^{\prime}$ and $b^{\prime}$ be the orthogonal projections of $S$ and $b$ onto $L^{\perp}$. Since $L$ is a lattice subspace, $S^{\prime}=\left(b^{\prime}+\Lambda\right) \cap \operatorname{proj}_{L^{\perp}}(\operatorname{conv}(S))$, where $\Lambda$ is a lattice in $L^{\perp}$. Since $\operatorname{proj}_{L^{\perp}}(K) \cap \operatorname{proj}_{L^{\perp}}(\operatorname{conv}(S))$ is a polytope, $\operatorname{proj}_{L^{\perp}}(K) \cap \operatorname{proj}_{L^{\perp}}(\operatorname{conv}(S)) \cap\left(b^{\prime}+\Lambda\right)$ is finite. Furthermore, since $\operatorname{proj}_{L^{\perp}}(S \cap K) \subseteq \operatorname{proj}_{L^{\perp}}(K) \cap \operatorname{proj}_{L^{\perp}}(\operatorname{conv}(S)) \cap\left(b^{\prime}+\Lambda\right)$, it follows that $\operatorname{proj}_{L^{\perp}}(S \cap K)$ is a finite set.

We conclude that the family of polyhedra $T(s), s \in S \cap K$, has a finite number of elements, thus $T(S, K)=\bigcup_{s \in S \cap K} T(s)$ is the union of a finite number of polyhedra.

\subsubsection{The covering property}

We assume here $S=\left(b+\mathbb{Z}^{n}\right) \cap Q$, where $Q$ is a rational polyhedron. As mentioned earlier, the minimal valid functions for $S$ are in one-to-one correspondence with maximal $S$-free convex sets containing the origin in their interior. For any such maximal $S$-free convex set $K$, we refer to the lifting region $T_{\psi}$ for the minimal cut-generating function $\psi$ corresponding to $K$ by $T(S, K)$, to emphasize the dependence on $S$ and $K$. We say $T(S, K)$ has the covering property if $T(S, K)+W_{S}=\mathbb{R}^{n}$. When $S$ is clear from the context, we will also say that $K$ has the covering property if $T(S, K)$ has the covering property.

Results of this section and their importance for discrete optimization. The main results presented in this section are three operations that preserve the covering property, namely, translations, the so-called coproduct and limit operations. Moreover, a necessary and sufficient condition is presented in Theorem 6.9 to characterize which pyramids in a particular family have the covering property.

The importance of these results in terms of cutting planes is the following. The pyramids in Theorem 6.9 and classification of the covering property in $\mathbb{R}^{2}$ (see [25]) provide a "base set" of maximal $S$-free convex sets with the covering property. By iteratively applying the three operations of translations, coproducts and limits, we can then build a vast (infinite) list of maximal $S$-free convex sets (in arbitrarily high dimensions) with the covering property, enlarging this "base set". Not only does this recover all the previously known sets with the covering property, it vastly expands this list. Earlier, ad hoc families of $S$-free convex sets were proven to have the covering property - now we have generic operations to construct infinitely many families. These make a contribution in the modern thrust on obtaining efficiently computable formulas for computing cutting planes, by giving a much wider class of maximal $S$-free sets whose lifting regions have the covering property.

The covering property is preserved under translations. Let $K$ be a maximal $S$-free polyhedron with the origin in its interior. In [25], Basu and Paat prove the following theorem.

TheOREM 6.7. Let $t \in \mathbb{R}^{n}$ be such that $K+t$ also contains the origin in its interior. Then, $T(S, K)+W_{S}=\mathbb{R}^{n}$ if and only if $T(S+t, K+t)+W_{S+t}=\mathbb{R}^{n}$. 
In other words, the covering property is preserved under translations.

This theorem is not obvious, since both the function $\psi$ defined according to (4.4) and the set $T(S, K)$ change in a non-trivial way when we translate $S$ and $K$. For the case $S=b+\mathbb{Z}^{n}$, this result was first proved when $K$ is a maximal $S$-free simplicial polytope [19] and then for any maximal $S$-free polyhedron in [9]. Both proofs are based on volume arguments, which do not seem easily extendable to the more general case $S=\left(b+\mathbb{Z}^{n}\right) \cap Q$ for a rational polyhedron $Q$. The generalization to this setting was obtained in [25] by using the invariance of domain as the main tool. This is an important result in algebraic topology, first proved by Brouwer [28, 46].

Theorem 6.8 (Invariance of Domain). If $U$ is an open subset of $\mathbb{R}^{n}$ and $f: U \rightarrow \mathbb{R}^{n}$ is an injective, continuous map, then $f(U)$ is open and $f$ is a homeomorphism between $U$ and $f(U)$.

An outline of the proof of the invariance of the lifting region under translations when $S=\left(b+\mathbb{Z}^{n}\right) \cap Q$ (Theorem 6.7) is now provided. We define $S^{\prime}=S+t$ and $K^{\prime}=K+t$.

1. Let $\left\{x \in \mathbb{R}^{n}: a_{i} x \leq 1, i \in I\right\}$ be an irredundant description of a maximal $S$-free polyhedron $K$ with $0 \in \operatorname{int}(K)$. For each $k \in I$, define the affine function $f_{k}$ that maps the affine hyperplane $H=\left\{r \in \mathbb{R}^{n}: a_{k} r=1\right\}$ to $H+t$.

2. For every $s \in K \cap S$ and $w \in W_{S}$, define the polyhedron $K_{s, w}=T(s)+w$ and define the map $f_{s, w}: K_{s, w} \rightarrow \mathbb{R}^{n}$ as $f_{s, w}(x)=f_{k(s)}(x-w)+w$, where $k(s) \in I$ is such that $a_{k(s)} s=1$. Since $T(S, K)+\mathbb{Z}^{n}=\mathbb{R}^{n}$, we have

$$
\bigcup_{s \in K \cap S, w \in W_{S}} K_{s, w}=T(S, K)+\mathbb{Z}^{n}=\mathbb{R}^{n}
$$

3. It is shown that for any two pairs $s_{1}, w_{1}$ and $s_{2}, w_{2}$ we have that $f_{s_{1}, w_{1}}(x)=f_{s_{2}, w_{2}}(x)$ for all $x \in K_{s_{1}, w_{1}} \cap K_{s_{2}, w_{2}}$. Thus, the different $f_{s, w}$ 's can be "stitched together" to give a well-defined map $f: \mathbb{R}^{n} \rightarrow \mathbb{R}^{n}$ such that $f$ restricted to $K_{s, w}$ is equal to $f_{s, w}$. Since each $f_{s, w}$ is an invertible affine map on $K_{s, w}$, and any bounded set intersects only finitely many polyhedra $K_{s, w}$, it can be shown that $f$ is an injective continuous map on $\mathbb{R}^{n}$.

4. It is also shown that $f_{s, w}(T(s)+w)=T(s+t)+w$ for every $s \in S \cap K$ and $w \in W_{S}$. In other words, the affine function $f_{s, w}$ maps a spindle in $T(S, K)$ (translated by the vector $w$ ) into the corresponding spindle in $T\left(S^{\prime}, K^{\prime}\right)$ (translated by the same vector $w)$.

5. By Theorem 6.8, $f\left(\mathbb{R}^{n}\right)$ is open. On the other hand, $f\left(\mathbb{R}^{n}\right)=T\left(S^{\prime}, K^{\prime}\right)+W_{S}$ can be shown to be closed because $T\left(S^{\prime}, K^{\prime}\right)$ is the union of finite many polyhedra translated by a lattice $W_{S}=\operatorname{lin}(\operatorname{conv}(S)) \cap \mathbb{Z}^{n}$. Since $\mathbb{R}^{n}$ is connected, the only non-empty closed and open subset of $\mathbb{R}^{n}$ is $\mathbb{R}^{n}$ itself. Thus, $f\left(\mathbb{R}^{n}\right)=\mathbb{R}^{n}$. 
6. Finally one observes that $W_{S}=\operatorname{lin}(\operatorname{conv}(S)) \cap \mathbb{Z}^{n}=W_{S^{\prime}}$. Therefore,

$$
\begin{aligned}
T\left(S^{\prime}, K^{\prime}\right)+W_{S^{\prime}} & =T\left(S^{\prime}, K^{\prime}\right)+W_{S} \\
& =\bigcup_{s^{\prime} \in K^{\prime} \cap S^{\prime}, w \in W_{S}} T\left(s^{\prime}\right)+w \\
& =\bigcup_{s \in K \cap S, w \in W_{S}} T(s+t)+w \\
& =\bigcup_{s \in K \cap S, w \in W_{S}} f_{s, w}(T(s)+w) \\
& =f\left(\bigcup_{s \in K \cap S, w \in W_{S}}(T(s)+w)\right) \\
& =f\left(T(S, K)+W_{S}\right) \\
& =f\left(\mathbb{R}^{n}\right) \\
& =\mathbb{R}^{n}
\end{aligned}
$$

where the fourth equality follows from Step 3.

The coproduct and limit operations preserve the covering property. We define an operation on polytopes that preserves the covering property. Namely, given two polytopes $K_{1} \in \mathbb{R}^{n_{1}}$ and $K_{2} \in \mathbb{R}^{n_{2}}$ with $K_{i}$ containing the origin in its interior for $i=1,2$, we define the coproduct $K_{1} \diamond K_{2}$ as follows. Let $K_{i}^{\bullet}$ be the (inclusion-wise) smallest prepolar for $K_{i}$, $i=1,2$. Define $K_{1} \diamond K_{2}=\left(K_{1}^{\bullet} \times K_{2}^{\bullet}\right)^{\circ}$ where we remind the reader that $V^{\circ}$ denotes the polar of a set $V$, and $X \times Y$ denotes the cartesian product of $X$ and $Y$. Let $n=n_{1}+n_{2}$ and for $i \in\{1,2\}$, let $S_{i}=\left(b_{i}+\mathbb{Z}^{n_{i}}\right) \cap Q_{i}$, where $Q_{i} \subseteq \mathbb{R}^{n_{i}}$ is a rational polyhedron and $b_{i} \in \mathbb{R}^{n_{i}} \backslash \mathbb{Z}^{n_{i}}$. Then $S_{1} \times S_{2}=\left(\left(b_{1}, b_{2}\right)+\left(\mathbb{Z}^{n_{1}} \times \mathbb{Z}^{n_{2}}\right)\right) \cap\left(Q_{1} \times Q_{2}\right)$. Therefore, it is reasonable to speak of $S_{1} \times S_{2}$-free convex sets. If $K_{i}$ is maximal $S_{i}$-free such that $T\left(S_{i}, K_{i}\right)$ has the covering property for $i \in\{1,2\}$, then for any $0 \leq \mu \leq 1, \frac{K_{1}}{\mu} \diamond \frac{K_{2}}{1-\mu}$ is maximal $S_{1} \times S_{2}$-free and $T\left(S_{1} \times S_{2}, \frac{K_{1}}{\mu} \diamond \frac{K_{2}}{1-\mu}\right)$ has the covering property. This is proved in [25]; it was first shown in [9] for the case when $S_{i}=b_{i}+\mathbb{Z}^{n_{i}}$ for $i \in\{1,2\}$. This is a very useful operation to create higher dimensional maximal $S$-free convex sets with the covering property by "gluing" together lower dimensional such sets.

When $S=\left(b+\mathbb{Z}^{n}\right) \cap Q$ for some rational polyhedron $Q$, it is shown in [25] that if a sequence of maximal $S$-free convex sets, all of whose lifting regions have the covering property, converges (in some precise sense) to a maximal $S$-free convex set, then the limit set also has the covering property. This is a generalization of a result from [9].

Special polytopes that have the covering property. In this last part we assume $S=b+\mathbb{Z}^{n}$. We define a pyramid as the convex hull of an $(n-1)$-dimensional polytope $B$ and a point $v \notin \operatorname{aff}(B) . v$ is called the apex and $B$ is the base of the pyramid.

Theorem 6.9. [9] Assume $S=b+\mathbb{Z}^{n}$ for some $b \notin \mathbb{Z}^{n}$, and let $K$ be a maximal $S$-free pyramid in $\mathbb{R}^{n}(n \geq 2)$ such that every facet of $K$ contains exactly one point from $S$ in its relative interior. $K$ has the covering property if and only if $K$ is the image of $\operatorname{conv}\left\{0, n e^{1}, \ldots, n e^{n}\right\}$ under an affine unimodular transformation.

Assume first that $K$ is $\operatorname{conv}\left\{0, n e^{1}, \ldots, n e^{n}\right\}$ (after applying an affine unimodular transformation). For each $i=1, \ldots, n$, consider the translation $K-n e^{i}$ and the spindle formed by the facet containing the point $e^{i}-n e^{i}$; this spindle is the unimodular transformation of the cube $[0,1]^{n}$. Moreover, the spindle in $K$ with respect to the point $(1,1, \ldots, 1)$ is the cube $[0,1]^{n}$. Thus, each of these translation vectors leads to a lifting region which covers $\mathbb{R}^{n}$ by 
integer translates. An extension of the translation invariance property can be used to show that this implies $K$ has the covering property.

We now outline the proof of the other implication.

1. Consider a translation of $K$ such that the apex of $K$ becomes the origin, and let $T$ be the spindle corresponding to the single integer point on the base of the pyramid. Using an extension of the translation invariance property proved above, one can show that $T$ covers $\mathbb{R}^{n}$ by integer translates. It is also not hard to show that two integer translates of $T$ cannot intersect in the interior. Thus $T$ actually tiles $\mathbb{R}^{n}$ by integer translates.

2. For a polytope $K$ and any face $F$ of $K$ of dimension $n-2$, the belt corresponding to $F$ in $K$ is the set of all facets that contain a translate of $F$ or $-F$. The Venkov-AlexandrovMcMullen theorem from the geometry of numbers states:

TheORem 6.10 ([56, Theorem 32.2]). Let $K$ be a compact convex set with nonempty interior that translatively tiles $\mathbb{R}^{n}$. Then the following assertions hold:

(a) $K$ is a centrally symmetric polytope.

(b) All facets of $K$ are centrally symmetric.

(c) Every belt of $K$ is either of length 4 or 6 .

This implies that $T$ is centrally symmetric with centrally symmetric facets.

3. It can be shown that since $T$, in this special case, is a spindle with centrally symmetric facets, every belt of $T$ is of length 4 . This, in turn, can be used to show that every face of dimension $n-2$ is centrally symmetric. McMullen's characterization of zonotopes [64] then implies that $T$ is a zonotope. (A zonotope is the Minkowski sum of finitely many line segments; equivalently, a zonotope is the image under an affine map - not necessarily invertible - of a cube.)

4. Combinatorial geometry of zonotopes can be used to show that any zonotope whose belts have all length 4 is a parallelotope (i.e., the invertible affine image of a cube). Thus $T$ is a parallelotope and $K$ is a simplex.

5. Since $T$ tiles $\mathbb{R}^{n}$ by integer translates, $T$ has volume 1 . Moreover, it can be shown that there exists a translation vector $t \in \mathbb{R}^{n}$ such that the polytope $2 T+t$ is centrally symmetric about the origin and the only integer point in its interior is the origin. Moreover, each facet of $2 T+t$ contains exactly one integer point in its relative interior. We now appeal to the Minkowski-Hajós theorem:

Theorem 6.11 ([57], Section 12.4, Chapter 2). Let $S$ be a 0 -symmetric parallelotope such that each it has no integer point in its interior besides 0 , and suppose that $S$ has volume $2^{n}$. Then there exists a unimodular transformation $U$ such that after applying $U, S$ will have two parallel facets given by $-1 \leq x_{1} \leq 1$.

We use this theorem to prove the following lemma. 
LEMma 6.12. Let $S$ be a 0-symmetric parallelotope with no integer point in its interior besides 0 , and suppose that $S$ has volume $2^{n}$. If every facet of $S$ has exactly one integer point in its relative interior, then $S$ is a unimodular transformation of the cube $[-1,1]^{n}$.

Proof. We prove this by induction on the dimension $n$. For $n=1$, this is trivial. Consider $n \geq 2$. The Minkowski-Hajós theorem (Theorem 6.11) implies that we can apply a unimodular transformation such that $S=\operatorname{conv}\left\{\left(S \cap\left\{x_{1}=-1\right\}\right) \cup\left(S \cap\left\{x_{1}=\right.\right.\right.$ $1\})\}$. Note that $S \cap\left\{x_{1}=-1\right\}, S \cap\left\{x_{1}=0\right\}$ and $S \cap\left\{x_{1}=1\right\}$ are all translations of each other. Therefore, $2^{n}=\operatorname{vol}(S)=2 \operatorname{vol}\left(S \cap\left\{x_{1}=0\right\}\right.$ ) (here we measure volume of $S \cap\left\{x_{1}=0\right\}$ in the $(n-1)$-dimensional linear space $\left.x_{1}=0\right)$. So $S \cap\left\{x_{1}=0\right\}$ has volume $2^{n-1}$. Therefore, $S \cap\left\{x_{1}=0\right\}$ is also a 0 -symmetric parallelotope in the linear space $x_{1}=0$ with volume $2^{n-1}$, and its only integer point is the origin. If any facet $F$ of $S \cap\left\{x_{1}=0\right\}$ contains two or more integer points in its relative interior, then the facet of $S$ passing through $F$ will contain these integer points in its relative interior, in contradiction to the hypothesis of the theorem. Therefore, every facet of $S \cap\left\{x_{1}=0\right\}$ contains at most one integer point in its relative interior. By the induction hypothesis, $S \cap\left\{x_{1}=0\right\}$ is equivalent to the cube $\left\{-1 \leq x_{i} \leq 1, i=2, \ldots, n\right\} \cap\left\{x_{1}=0\right\}$. Recall that $S \cap\left\{x_{1}=-1\right\}$ and $S \cap\left\{x_{1}=1\right\}$ are translations of $S \cap\left\{x_{1}=0\right\}$. Since $S \cap\left\{x_{1}=0\right\}$ is equivalent to the cube $\left\{-1 \leq x_{i} \leq 1, i=2, \ldots, n\right\} \cap\left\{x_{1}=0\right\}$, any translation by a non-integer vector $\left(x_{1}, x_{2}, \ldots, x_{n}\right)$ with $x_{1} \in \mathbb{Z}$ will contain at least two integer points in its relative interior. But the facets $S \cap\left\{x_{1}=-1\right\}$ and $S \cap\left\{x_{1}=1\right\}$ contain at most one integer point in their relative interior. Therefore, they are in fact integer translates of $S \cap\left\{x_{1}=0\right\}$. This proves the lemma.

We know that $2 T+t$ satisfies the hypothesis of Lemma 6.12 and therefore $2 T+t$ is a unimodular transformation of the cube $[-1,1]^{n}$. This can be used to show that $K$ is the image of $\operatorname{conv}\left\{0, n e^{1}, \ldots, n e^{n}\right\}$ under an affine unimodular transformation.

For further details, we refer the reader to [9, Section 6] and [19, Section 3].

\section{References}

[1] János Aczél and Jean G Dhombres. Functional Equations in Several Variables. Number 31 in Encyclopedia of Mathematics and its Applications. Cambridge university press, 1989 .

[2] Ravi P. Agarwal, Maria Meehan, and Donal O'Regan. Fixed Point Theory and Applications. Cambridge University Press, 2001.

[3] Iskander Aliev, Robert Bassett, Jesús A De Loera, and Quentin Louveaux. A quantitative doignon-bell-scarf theorem. Combinatorica, pages 1-20, 2016.

[4] Kent Andersen, Quentin Louveaux, Robert Weismantel, and Laurence Wolsey. Inequalities from two rows of a simplex tableau. In Matteo Fischetti and David Williamson, editors, Integer Programming and Combinatorial Optimization. 12th International IPCO Conference, Ithaca, NY, USA, June 25-27, 200\%. Proceedings, volume 4513 of Lecture Notes in Computer Science, pages 1-15. Springer Berlin / Heidelberg, 2007. 
[5] Alper Atamtürk and Vishnu Narayanan. Conic mixed-integer rounding cuts. Math. Program., 122(1):1-20, 2010.

[6] Alper Atamtürk and Vishnu Narayanan. Lifting for conic mixed-integer programming. Math. Program., 126(2):351-363, 2011.

[7] Gennadiy Averkov. On maximal S-free sets and the helly number for the family of s-convex sets. SIAM Journal on Discrete Mathematics, 27(3):1610-1624, 2013.

[8] Gennadiy Averkov. A proof of Lovász's theorem on maximal lattice-free sets. Beitr. Algebra Geom., 54(1):105-109, 2013.

[9] Gennadiy Averkov and Amitabh Basu. Lifting properties of maximal lattice-free polyhedra. http://arxiv.org/abs/1404.7421.

[10] Gennadiy Averkov, Christian Wagner, and Robert Weismantel. Maximal lattice-free polyhedra: finiteness and an explicit description in dimension three. Math. Oper. Res., 36(4):721-742, 2011.

[11] Egon Balas. Intersection cuts - a new type of cutting planes for integer programming. Operations Research, 19:19-39, 1971.

[12] Egon Balas, Sebastián Ceria, Gérard Cornuéjols, and Nellisery R. Natraj. Gomory cuts revisited. Operations Research Letters, 19(1):1-9, 1996.

[13] Egon Balas and Robert G. Jeroslow. Strengthening cuts for mixed integer programs. European Journal of Operational Research, 4(4):224-234, 1980.

[14] A. Barvinok. A course in convexity. American Mathematical Society, 2002.

[15] Amitabh Basu, Pierre Bonami, Gérard Cornuéjols, and François Margot. Experiments with two-row cuts from degenerate tableaux. INFORMS Journal on Computing, 23(4):578-590, 2011.

[16] Amitabh Basu, Manoel Campêlo, Michele Conforti, Gérard Cornuéjols, and Giacomo Zambelli. Unique lifting of integer variables in minimal inequalities. Mathematical Programming, 141(1-2, Ser. A):561-576, 2013.

[17] Amitabh Basu, Michele Conforti, Gérard Cornuéjols, and Giacomo Zambelli. Maximal lattice-free convex sets in linear subspaces. Mathematics of Operations Research, 35:704$720,2010$.

[18] Amitabh Basu, Michele Conforti, Gérard Cornuéjols, and Giacomo Zambelli. Minimal inequalities for an infinite relaxation of integer programs. SIAM Journal on Discrete Mathematics, 24:158-168, February 2010.

[19] Amitabh Basu, Gérard Cornuéjols, and Matthias Köppe. Unique minimal liftings for simplicial polytopes. Mathematics of Operations Research, 37(2):346-355, 2012.

[20] Amitabh Basu, Gérard Cornuéjols, and Giacomo Zambelli. Convex sets and minimal sublinear functions. Journal of Convex Analysis, 18:427-432, 2011. 
[21] Amitabh Basu, Robert Hildebrand, and Matthias Köppe. Equivariant perturbation in Gomory and Johnson's infinite group problem. III. Foundations for the $k$-dimensional case and applications to $k=2$. eprint arXiv:1403.4628 [math.OC], 2014.

[22] Amitabh Basu, Robert Hildebrand, and Matthias Köppe. Light on the infinite group problem. eprint http://arxiv.org/abs/1410.8584, 2014.

[23] Amitabh Basu, Robert Hildebrand, and Matthias Köppe. Equivariant perturbation in Gomory and Johnson's infinite group problem. I. The one-dimensional case. Mathematics of Operations Research, 40(1):105-129, 2015.

[24] Amitabh Basu, Robert Hildebrand, Matthias Köppe, and Marco Molinaro. A ( $k+$ 1 )-slope theorem for the $k$-dimensional infinite group relaxation. SIAM Journal on Optimization, 23(2):1021-1040, 2013.

[25] Amitabh Basu and Joe Paat. Operations that preserve the covering property of the lifting region. SIAM Journal on Optimization, 25(4):2313-2333, 2015.

[26] David E Bell. A theorem concerning the integer lattice. Studies in Applied Mathematics, 56(2):187-188, 1977.

[27] Valentin Borozan and Gérard Cornuéjols. Minimal valid inequalities for integer constraints. Mathematics of Operations Research, 34:538-546, 2009.

[28] Luitzen EJ Brouwer. Beweis der invarianz desn-dimensionalen gebiets. Mathematische Annalen, 71(3):305-313, 1911.

[29] Sebastián Ceria and João Soares. Convex programming for disjunctive convex optimization. Mathematical Programming, 86(3):595-614, 1999.

[30] Mehmet T. Çezik and Garud Iyengar. Cuts for mixed 0-1 conic programming. Math. Program., 104(1):179-202, September 2005.

[31] Michele Conforti, Gérard Cornuéjols, Aris Daniilidis, Claude Lemaréchal, and Jérôme Malick. Cut-generating functions. In Integer Programming and Combinatorial Optimization, pages 123-132. Springer, 2013.

[32] Michele Conforti, Gérard Cornuéjols, and Giacomo Zambelli. Corner polyhedra and intersection cuts. Surveys in Operations Research and Management Science, 16:105$120,2011$.

[33] Michele Conforti, Gérard Cornuéjols, and Giacomo Zambelli. A geometric perspective on lifting. Oper. Res., 59(3):569-577, 2011.

[34] Michele Conforti, Gérard Cornuéjols, and Giacomo Zambelli. Integer programming, volume 271. Springer, 2014.

[35] Gérard Cornuéjols. Revival of the gomory cuts in the 1990?s. Annals of Operations Research, 149(1):63-66, 2007. 
[36] Gérard Cornuéjols and François Margot. On the facets of mixed integer programs with two integer variables and two constraints. Mathematical Programming, 120:429-456, 2009 .

[37] Gérard Cornuéjols and Marco Molinaro. A 3-Slope Theorem for the infinite relaxation in the plane. Mathematical Programming, 142(1-2):83-105, 2013.

[38] Gérard Cornuéjols, Laurence Wolsey, and Sercan Yıldız. Sufficiency of cut-generating functions. Mathematical Programming, 152(1-2):643-651, 2015.

[39] Alberto Del Pia and Robert Weismantel. Relaxations of mixed integer sets from latticefree polyhedra. $4 O R, 10(3): 221-244,2012$.

[40] Santanu S Dey, Andrea Lodi, Andrea Tramontani, and Laurence A Wolsey. Experiments with two row tableau cuts. In Integer Programming and Combinatorial Optimization, pages 424-437. Springer, 2010.

[41] Santanu S. Dey and Jean-Philippe P. Richard. Facets of two-dimensional infinite group problems. Mathematics of Operations Research, 33(1):140-166, 2008.

[42] Santanu S. Dey, Jean-Philippe P. Richard, Yanjun Li, and Lisa A. Miller. On the extreme inequalities of infinite group problems. Mathematical Programming, 121(1):145-170, June 2009.

[43] Santanu S. Dey and Laurence A. Wolsey. Constrained infinite group relaxations of mips. SIAM Journal on Optimization, 20(6):2890-2912, 2010.

[44] Santanu S. Dey and Laurence A. Wolsey. Two row mixed-integer cuts via lifting. Mathematical Programming, 124(1-2):143-174, 2010.

[45] J.-P. Doignon. Convexity in cristallographical lattices. J. Geometry, 3:71-85, 1973.

[46] Albrecht Dold. Lectures on Algebraic Topology. Springer-Verlag, Berlin/Heidelberg, Germany, 1995.

[47] R. E. Gomory. Outline of an algorithm for integer solutions to linear programs. Bull. Amer. Math. Soc., 64:275-278, 1958.

[48] R. E. Gomory. On the relation between integer and noninteger solutions to linear programs. Proc. Nat. Acad. Sci. U.S.A., 53:260-265, 1965.

[49] Ralph Gomory. An algorithm for the mixed integer problem. Technical report, DTIC Document, 1960.

[50] Ralph E. Gomory. Some polyhedra related to combinatorial problems. Linear Algebra and its Applications, 2(4):451-558, 1969.

[51] Ralph E Gomory. The atoms of integer programming. Annals of Operations Research, 149(1):99-102, 2007. 
[52] Ralph E. Gomory and Ellis L. Johnson. Some continuous functions related to corner polyhedra, I. Mathematical Programming, 3:23-85, 1972.

[53] Ralph E. Gomory and Ellis L. Johnson. Some continuous functions related to corner polyhedra, II. Mathematical Programming, 3:359-389, 1972.

[54] Ralph E. Gomory and Ellis L. Johnson. T-space and cutting planes. Mathematical Programming, 96:341-375, 2003.

[55] R.E. Gomory. An algorithm for integer solutions to linear programs. In Recent advances in mathematical programming, pages 269-302. McGraw-Hill, New York, 1963.

[56] P. M. Gruber and C. G. Lekkerkerker. Geometry of Numbers. North-Holland, 1987.

[57] P. M. Gruber and C. G. Lekkerkerker. Geometry of numbers, volume 37 of North-Holland Mathematical Library. North-Holland Publishing Co., Amsterdam, second edition, 1987.

[58] Ellis L. Johnson. On the group problem for mixed integer programming. Mathematical Programming Study, 2:137-179, 1974.

[59] Ellis L Johnson. Characterization of facets for multiple right-hand choice linear programs. In Mathematical Programming at Oberwolfach, pages 112-142. Springer, 1981.

[60] B. Knaster, C. Kuratowski, and S. Mazurkiewicz. Ein Beweis des Fixpunktsatzes für n-dimensionale Simplexe. Fundamenta Mathematicae, 14:132-137, 1929.

[61] C Lemaréchal and JB Hiriart-Urruty. Convex analysis and minimization algorithms I. Grundlehren der mathematischen Wissenschaften, 305, 1996.

[62] Quentin Louveaux and Laurent Poirrier. An algorithm for the separation of two-row cuts. Mathematical Programming, 143(1-2):111-146, 2014.

[63] László Lovász. Geometry of numbers and integer programming. In M. Iri and K. Tanabe, editors, Mathematical Programming: State of the Art, pages 177-201. Mathematical Programming Society, 1989.

[64] P. McMullen. Polytopes with centrally symmetric faces. Israel J. Math., 8:194-196, 1970.

[65] Diego A Morán R and Santanu S Dey. On maximal S-free convex sets. SIAM Journal on Discrete Mathematics, 25(1):379, 2011.

[66] Jean-Philippe P. Richard and Santanu S. Dey. The group-theoretic approach in mixed integer programming. In Michael Jünger, Thomas M. Liebling, Denis Naddef, George L. Nemhauser, William R. Pulleyblank, Gerhard Reinelt, Giovanni Rinaldi, and Laurence A. Wolsey, editors, 50 Years of Integer Programming 1958-2008, pages 727-801. Springer Berlin Heidelberg, 2010.

[67] R. T. Rockafellar. Convex Analysis. Princeton University Press, Princeton, New Jersey, 1970. 
[68] Herbert E Scarf. An observation on the structure of production sets with indivisibilities. Proceedings of the National Academy of Sciences, 74(9):3637-3641, 1977.

[69] Robert A Stubbs and Sanjay Mehrotra. A branch-and-cut method for 0-1 mixed convex programming. Mathematical Programming, 86(3):515-532, 1999.

[70] Sercan Yıldız and Gérard Cornuéjols. Cut-generating functions for integer variables. Mathematics of Operations Research, 41:1381-1403, 2016.

[71] Giacomo Zambelli. On degenerate multi-row Gomory cuts. Operations Research Letters, 37(1):21-22, January 2009.

[72] G. M. Ziegler. Lectures on Polytopes, volume 152 of Graduate Texts in Mathematics. Springer-Verlag, New York, 1995. 